\title{
Identification of a prognostic signature of nine metabolism- related genes for hepatocellular carcinoma
}

\author{
Chaozhi Tang Equal first author, 1 , Jiakang Ma ${ }^{\text {Equal first author, } 2, \text { Xiuli Liu }}{ }^{3}$, Zhengchun Liu ${ }^{\text {Corresp. } 4}$ \\ ${ }^{1}$ Department of Urology, The First Affiliated Hospital of China Medical University, Shenyang, Liaoning, China \\ 2 Department of Oncology, The Second Affiliated Hospital of Zhengzhou University, Zhengzhou, Henan, China \\ 3 Department of Oncology, Affiliated Hospital of Guilin Medical University, Guilin, Guangxi, China \\ 4 Department of Radiation Oncology, Affiliated Hospital of Guilin Medical University, Guilin, Guangxi, China \\ Corresponding Author: Zhengchun Liu \\ Email address: 18624879717@163.com
}

Background. Hepatocellular carcinoma (HCC) is the fifth most common cancer. Since changes in liver metabolism contribute to liver disease development, it is necessary to build a metabolism-related prognostic model for HCC.

Methods. We constructed a metabolism-related-gene (MRG) signature comprising nine genes, which segregated HCC patients into high- and low-risk groups.

Results. The survival rate (overall survival: OS; relapse-free survival; and progression-free survival) of patients in the low-risk group of The Cancer Genome Atlas (TCGA) cohort was significantly higher than that of patients in the high-risk group. The OS prognostic signature was validated in the International Cancer Genome Consortium independent cohort. The corresponding receiver operating characteristic curves of the model indicated that the signature had good diagnostic efficiency, in terms of improving OS over 1, 3, and 5 years. Hierarchical analysis demonstrated that the MRG signature was significantly associated with better prognosis in male patients, patients aged $\leq 65$ years, and patients carrying the wild-type TP53 or CTNNB1 genes. A nomogram was established, and good performance and clinical practicability were confirmed. Additionally, using the GSE109211 dataset from the Gene Expression Omnibus database, we were able to verify that the nine genes in this MRG signature had different responses to sorafenib, suggesting that some of these MRGs may act as therapeutic targets for HCC.

Conclusions. We believe that these findings will add value in terms of the diagnosis, treatment, and prognosis of HCC. 


\section{Identification of a Prognostic Signature of Nine}

3 Metabolism-related Genes for Hepatocellular

4 Carcinoma

5

${ }^{1}$ Department of Urology, The First Affiliated Hospital of China Medical University, Shenyang, Liaoning, China

${ }^{2}$ Department of Oncology, The Second Affiliated Hospital of Zhengzhou University,

11 Zhengzhou, Henan, China

${ }^{3}$ Department of Oncology, Affiliated Hospital of Guilin Medical University, Guilin, Guangxi, China

${ }^{4}$ Department of Radiation Oncology, Affiliated Hospital of Guilin Medical University, Guilin, Guangxi, China

Corresponding Author:

Zhengchun Liu*

Department of Radiation Oncology, Affiliated Hospital of Guilin Medical University, Renmin Rd, Lingui District, Guilin, Guangxi, China

Email address: 18624879717@163.com

\section{Abstract}

Background. Hepatocellular carcinoma (HCC) is the fifth most common cancer. Since changes in liver metabolism contribute to liver disease development, it is necessary to build a metabolism-related prognostic model for HCC.

Methods. We identified a metabolism-related gene (MRG) signature comprising nine genes, which segregated HCC patients into high- and low-risk groups.

Results. The survival rate (overall survival [OS], relapse-free survival, and progression-free survival) of patients in the low-risk group of The Cancer Genome Atlas (TCGA) cohort was significantly higher than that in the high-risk group. The OS prognostic signature was validated in the International Cancer Genome Consortium independent cohort. The corresponding receiver operating characteristic curves of the model indicated that the signature had good diagnostic efficiency in terms of improving OS over periods of 1,3, and 5 years. Hierarchical analysis demonstrated that the MRG signature was significantly associated with better prognosis in male patients, patients aged $\leq 65$ years, and patients carrying the wild-type TP53 or CTNNB1 genes. A nomogram was established, and good performance and clinical practicability were confirmed. Additionally, using the GSE109211 dataset from the Gene Expression Omnibus database, we 
39 were able to verify that the nine MRGs in this signature showed different responses to sorafenib,

40 which suggested that some of them may serve as therapeutic targets for HCC.

41 Conclusions. These findings will add value in terms of the diagnosis, treatment, and prognosis

42 of HCC.

43 Introduction

44 Hepatocellular carcinoma (HCC) is the fifth most common cancer and the most common type of

45 hepatobiliary cancer, with more than 500,000 new cases diagnosed each year and an annual

46 mortality rate of 250,000 (Schlachterman et al. 2015; Singal \& El-Serag 2015; Steel et al. 2007).

47 In North America and several European regions, the incidence and mortality rates of HCC have

48 been rising, and in the United States alone, the incidence of HCC has more than doubled in the

49 past 20 years (Singal \& El-Serag 2015). Currently, only $46 \%$ of HCC cases are diagnosed early,

50 with most cases being diagnosed too late to allow for successful treatment (Njei et al. 2015). The

51 5-year overall survival (OS) of all stages of HCC is $12 \%$, increasingly making it the most

52 important cause of cancer-related death (Hilmi et al. 2019). In modern clinical practice, the

53 major risk factors for $\mathrm{HCC}$ are chronic hepatitis $\mathrm{C}$ virus (HCV) or hepatitis B virus (HBV)

54 infection, heavy drinking, diabetes, and nonalcoholic fatty liver (Budny et al. 2017; Hsu et al.

55 2015; Levrero \& Zucman-Rossi 2016). In particular, active HCV and HBV contribute the most

56 to the burden of global HCC. Several advances related to HCC prevention and early detection

57 and diagnosis have proven to be effective and have led to a reduction in the incidence of HCC

58 and the mortality associated with it. Nonetheless, there are several common challenges in

59 detecting and treating HCC, including limited awareness about high-risk patients, limited

60 availability of effective and validated risk-stratification measures, and high costs of monitoring

61 at-risk populations.

$62 \mathrm{HCC}$ is a highly angiogenic solid tumour characterised by cell cycle disorders, abnormal

63 angiogenesis, and escape from apoptosis (Bhagyaraj et al. 2019; Rebouissou \& Nault 2020). The

64 molecular pathogenesis of HCC is complex and involves a variety of genetic and epigenetic

65 changes, chromosomal aberrations, genetic mutations, and altered molecular pathways (Farazi \&

66 DePinho 2006). Metabolic alteration is one of the important features of tumours. The liver is an

67 important hub for metabolism of the three major nutrients - sugar, lipid, and amino acids - in the

68 body. HCC exhibits a variety of characteristic metabolic changes, such as increased oxygen

69 glycolysis, de novo fat synthesis, glutamine consumption, and oxidative metabolic imbalance,

70 which provide energy to the rapidly growing and proliferating tumour cells. The process of

71 metabolic alterations in tumours is regulated by multiple factors, such as changes in metabolic

72 enzyme activity, abnormal gene expression, and dysfunctional signal transduction pathways.

73 Many clinical parameters currently used to assess liver function reflect changes in enzyme

74 activity and metabolites. In fact, the difference in glucose and acetate utilisation has been used as

75 an effective clinical tool to stratify HCC patients. In addition, elevated serum lactate levels can

76 distinguish HCC patients from healthy individuals, and serum lactate dehydrogenase is used as a

77 prognostic indicator for patients with HCC during treatment. It is thus necessary to build a

78 metabolism-related prognostic model for HCC (De Matteis et al. 2018; Nakagawa et al. 2018; 
79 Pope et al. 2019). Changes in metabolic pathways, which are driven by oncogenes, are

80

81

82

83

84

85

86

87

88

89

90

91

92

93

94

95

96

97

98

99

100

101

102

103

104

105

106

107

108

109

110

111

112

113

114

115

116

117

118 recognised as cancer markers, and such changes provide cancers with a selective advantage for tumour growth, proliferation, and metastasis (Berndt et al. 2019; De Matteis et al. 2018; Huang et al. 2014; Kim et al. 2019). TP53 and CTNNB1 are two genes that are most prone to mutations in $\mathrm{HCC}$, and have received continuous research attention because of their involvement in events that dominate tumour development and progression. (Calderaro et al. 2017; Cancer Genome Atlas Research Network. Electronic address \& Cancer Genome Atlas Research 2017; ZucmanRossi et al. 2015). Treatment with sorafenib, the first approved systemic therapeutic agent for HCC, has shown significant improvements in survival outcomes of HCC patients (Ogasawara et al. 2018; Pinter \& Peck-Radosavljevic 2018). It inhibits cell growth, induces apoptosis, and downregulates the anti-apoptotic protein Mcl-1 by targeting a variety of protein kinases (Tai et al. 2013).

In this study, we constructed a prognostic model based on a metabolism-related gene (MRG) signature comprising nine genes. Further, we examined the associations among this signature, sorafenib use, and common clinical characteristics such as sex, age, and the presence or absence of mutations in TP53 and CTNNB1. The findings from this study will supplement the existing literature on $\mathrm{HCC}$ by providing useful data regarding molecular diagnosis and precision treatment and will help in screening drug targets for HCC.

\section{Materials \& Methods}

\section{Data processing and extraction of differentially expressed MRGs}

For the discovery cohort ( $\mathrm{n}=374$ patients), fragments per kilobase of exon per million mapped reads (FPKM) data and the corresponding clinical information related to gene expression were downloaded from The Cancer Genome Atlas (TCGA). For the validation cohort $(\mathrm{n}=243$ patients), the original data and corresponding clinical data related to gene expression were downloaded from the Liver Cancer-RIKEN, Japan (LIRI-JP) project of the International Cancer Genome Consortium (ICGC). For analysis of OS, relapse-free survival (RFS), and progressionfree survival (PFS) in the discovery cohort, and that of OS in the validation cohort, we used data from 34, 301, 337, and 230 HCC patients, respectively; these patients had been followed up for 30 days or more, and patients who were likely to die of lethal complications (heart failure and haemorrhage) other than HCC were excluded from the study. The probes were annotated using Homo sapiens GTF files in the Ensembl database (http://asia.ensembl.org/index.html).

We selected all the pathways related to metabolism among 186 pathways from c2.cp.kegg.v7.0.symbols.gmt. In total, 944 MRGs were obtained, of which 918 MRGs were common between TCGA and ICGC. TCGA and ICGC MRG expression matrices were background-corrected using the R package "sva" with the "limma" package to identify differentially expressed MRGs. Genes having $|\log 2 \mathrm{FC}|>2$ and P-value $<0.05$ were selected for further analysis. The genes whose expression values were more than 0.5 and thus subjected to $\log 2$ transformation are depicted in a heat map. Finally, 54 differential MRGs were selected. The GSE109211 dataset was downloaded from the GEO database

(https://www.ncbi.nlm.nih.gov/geo/) and contained 140 cases of HCC (67 patients who received 
119 sorafenib and 73 patients who received placebo). This dataset was used for gene annotation

120 based on the GPL13938 platform (Illumina HumanHT-12 WG-DASL V4.0 expression

121 beadchip).

122 Identification of a prognostic signature based on differential MRG expression

123 After screening for differentially expressed MRGs, we performed univariate Cox regression

124 analysis to identify prognostic differentially expressed MRGs. A p-value $<0.05$ was considered

125 statistically significant. Next, the "glmnet" package was used to implement the LASSO Cox

126 regression model. All regression coefficients, including the coefficients of many unrelated

127 features, were reduced to zero in LASSO, precisely according to the adjustment weight $\lambda$. The

128 best $\lambda$ was selected based on the minimum cross-validation error. Finally, we calculated the risk

129 coefficients of the genes associated with the LASSO Cox regression model and estimated the

130 median risk, using the "survminer" package in R.

\section{Survival analysis}

132 The risk scores of HCC patients were calculated using the previously described formula, and the 133 patients were classified into high- and low-risk groups, according to the median. The Kaplan-

134 Meier plotter was utilised to estimate the difference in survival time between the high-and low-

135 risk patients. A p-value $<0.05$ for the log-rank test on both sides indicated a significant

136 difference in survival time between the two groups.

\section{Construction of nomogram}

138 We used the mutual clinical traits in the discovery and validation cohorts to construct a

139 nomogram, using the "rms" R package. We assessed the prognostic accuracy of the nomograms

140 by evaluating the corresponding calibration plots. The predicted and observed results of the

141 nomogram are shown in a calibration curve; the $45^{\circ}$ line indicated the best prediction.

142 Statistical analysis

143 A time-dependent receiver operating characteristic (ROC) analysis was performed to explore the

144 prognostic accuracy of the classifier, based on multiple differentially expressed MRGs, using the

145 "survivalROC" package in R. The Kaplan-Meier plotter was used to analyse the OS, RFS, and

146 PFS of the discovery cohort, and the OS of the validation cohort. Statistical differences between

147 the groups were evaluated using the log-rank test. Univariate and multivariate Cox regression

148 analyses were performed to assess the prognostic value of clinical characteristics and the risk

149 score, and clinical stratified analyses were performed to test whether the risk score was

150 independent of other clinical features, including age, sex, risk factors (HBV + HCV, alcohol

151 intake), degree of fibrosis (no fibrosis or fibrosis and cirrhosis), tumour grade (G), American

152 Joint Committee on Cancer (AJCC) stage, and tumour (T) stage, which were used as covariates.

153 Hazard ratios (HRs) and their respective 95\% confidence intervals (CI) were obtained. A p-value

$154<0.05$ was considered statistically significant. The statistical tests were bilateral and conducted

155 using R software (version 3.5.3)

156 Gene set enrichment analysis (GSEA)

157 Based on the median risk, 343 HCC samples from TCGA group and 230 HCC samples from the

158 ICGC group were divided into high- and low-risk groups. To identify metabolic and other 
159 important changes in the Kyoto Gene and Genome Encyclopaedia (KEGG) pathways, we used

160

161

162

163

164

165

166

167

168

169

170

171

172

173

174

175

176

177

178

179

180

181

182

183

184

185

186

187

188

189

190

191

192

193

194

195

196

197

198

GSEA version 4.0.1 to perform a GSEA between the high- and low-risk populations. The annotated gene set (c2.cp.kegg.v7.0.symbols.gmt) was defined as the reference gene set. False discovery rate $(\mathrm{FDR})<0.05$ was set as the cut-off.

\section{Results}

\section{Differential MRG expression}

MRG matrices, consisting of 50 normal and 374 HCC samples from TCGA and 202 normal and $243 \mathrm{HCC}$ samples from the ICGC, were obtained. Nine downregulated and 45 upregulated MRGs were identified. Univariate Cox regression analysis was used to identify prognostic MRGs.

\section{Identification of the signature based on multiple MRGs}

Using univariate Cox regression analysis, 22 MRGs closely associated with OS were identified (Supplementary Table 1). To prevent over-fitting of MRGs and to determine the best gene combination for the signature, we performed LASSO regression analysis. Finally, nine MRGs were identified to establish a prognostic model. We used the coefficients of the multivariate Cox regression model, which combined the predictions of the expression of the nine MRGs and their corresponding survival times and survival states to construct the following risk score formula: risk score $=(0.0193 \times R R M 2$ expression $)+(0.0068 \times D T Y M K$ expression $)+(0.0003 \times$ LPCAT1 expression $)+(-0.0013 \times L C A T$ expression $)+(0.0087 \times T X N R D 1$ expression $)+(0.0035 \times$ G6PD expression $)+(0.0012 \times P T G E S$ expression $)+(0.0508 \times$ ENTPD 2 expression $)+(0.0729$ $\times U C K 2$ expression) (Fig. 1). Among the nine prognostic MRGs, LCAT had a negative coefficient in Cox regression analysis, suggesting that its higher expression level was associated with lower risk and better OS. In contrast, RRM2, DTYMK, LPCAT1, TXNRD1, G6PD, PTGES, ENTPD2, and UCK2 showed positive coefficients; their elevated expression levels were accompanied by higher risk scores, thus predicting poor OS. According to the median risk score of 0.60 , patients were divided into high- and low-risk groups. A risk score greater than 0.60 was considered to indicate high risk, whereas a risk score $\leq 0.60$ was considered to indicate low risk. The accuracy of the OS predictions, based on the MRG signature, was evaluated by generating the ROC curves over time at 1,3, and 5 years. A higher area under curve (AUC) represents a better prognostic capability. The AUCs based on the MRG signatures in the discovery and validation cohorts were $0.797,0.703$, and 0.652 (in TCGA cohort), and $0.778,0.768$, and 0.835 (in the ICGC cohort), respectively (Fig. 2A and 2B). These results suggested that the prognostic model had high sensitivity and specificity. The results of the univariate and multivariate Cox analyses showed that the risk score remained an independent predictor, after adjusting for other covariates, in both the discovery and validation cohorts, and that gender and stage acted as independent predictors in the validation cohort (Fig. 2C-F).

\section{Prognostic value of the MRG signature}

To investigate the association between the MRG signature and prognosis, we performed an analysis of OS, RFS, and PFS in the high- and low-risk groups of the discovery cohort (Table 1), and that of OS in the high- and low-risk groups of the validation cohort (Table 2, Fig. 3). In the

Peer] reviewing PDF | (2020:04:47605:2:1:NEW 28 Jul 2020) 
199

200

201

202

203

204

205

206

207

208

209

210

211

212

213

214

215

216

217

218

219

220

221

222

223

224

225

226

227

228

229

230

231

232

233

234

235

236

237

238

discovery cohort, patients in the low-risk group had longer OS (HR $=2.20,95 \% \mathrm{CI}=1.51-3.19$, $\mathrm{p}<0.001)$, RFS $(\mathrm{HR}=1.62,95 \% \mathrm{CI}=1.16-2.27, \mathrm{p}=0.005)$, and $\mathrm{PFS}(\mathrm{HR}=1.64,95 \% \mathrm{CI}=$ $1.21-2.20, \mathrm{p}=0.001)$ than patients in the high-risk group. We also used the validation cohort to verify $\mathrm{OS}(\mathrm{HR}=5.22,95 \% \mathrm{CI}=2.81-9.68, \mathrm{p}<0.001)$. As predicted, patients in the high-risk group had higher mortality than patients in the low-risk group. Thus, the MRG signature can be considered an indicator of risk for HCC.

\section{Associations between the MRG signature and OS by sex and age}

To investigate the impact of clinical characteristics on the prognostic value of the MRG signature, we categorised HCC patients based on six clinical traits: age, sex, risk factors (HBV + $\mathrm{HCV}$, alcohol intake), tumour grade (G), AJCC stage, and tumour (T) stage. This stratified analysis revealed that OS, RFS, and PFS were significantly affected in male patients and those aged $\leq 65$ years in the discovery cohort; it also revealed significant OS results in the validation cohort (Fig. 4 and 5). Moreover, the OS, RFS, and PFS results for patients in the alcohol-intake, degree of fibrosis (no fibrosis or fibrosis and cirrhosis), G1-G2, and T1-T2 groups in the discovery cohort were also significant . However, the corresponding clinical information was not complete for the validation cohort. The prognostic stratified analysis showed that the survival rate of male patients in the low-risk group was significantly higher (OS $[\mathrm{HR}=2.85,95 \% \mathrm{CI}=$ $1.73-4.71, \mathrm{p}<0.001]$, RFS $[\mathrm{HR}=1.77,95 \% \mathrm{CI}=1.18-2.66, \mathrm{p}=0.006]$, and PFS $[\mathrm{HR}=1.76$, $95 \% \mathrm{CI}=1.21-2.55, \mathrm{p}=0.003])$ than that of patients in the high-risk (validation cohort) group (OS $[\mathrm{HR}=4.52,95 \% \mathrm{CI}=1.66-12.25, \mathrm{p}=0.003])$. In addition, the survival rate of patients aged $\leq 65$ years in the low-risk group was significantly higher $(\mathrm{OS}[\mathrm{HR}=1.97,95 \% \mathrm{CI}=1.21-3.21, \mathrm{p}$ $=0.007]$, RFS $[\mathrm{HR}=1.62,95 \% \mathrm{CI}=1.06-2.47, \mathrm{p}=0.027]$, and $\mathrm{PFS}[\mathrm{HR}=1.64,95 \% \mathrm{CI}=$ $1.13-2.39, \mathrm{p}=0.009])$ than that of patients in the high-risk (ICGC) group (OS $[\mathrm{HR}=5.58,95 \%$ $\mathrm{CI}=1.55-20.11, \mathrm{p}=0.009])$.

\section{Associations between the MRG signature and OS in patients carrying wild-type TP53 or}

\section{CTNNB1}

The tumour suppressor gene TP53 and the oncogene CTNNB1 are most commonly mutated in HCC and are associated with poor prognosis. Numerous studies, including epigenetic studies, have shown that mutations are important drivers of tumour carcinogenesis. Therefore, we performed a stratified analysis based on the mutation status of TP53 or CTNNB1 (Figure 6). For patients with wild-type $T P 53$ or $C T N N B 1$, the probability of death was significantly higher in the high-risk group than in the low-risk group. These results were consistent between the data from the two independent databases, TCGA and ICGC. Therefore, this MRG signature can be considered a risk indicator for patients carrying wild-type TP53 or CTNNB1.

\section{Independent data set drug trial validation using the GEO database}

To explore the association between the MRGs and drug response, we analysed differences in expression of the MRGs, using the GSE109211 dataset from the GEO database. This dataset comprised 67 patients treated with sorafenib (46 non-responders, 21 responders) and 73 patients who received placebo treatment (Fig. 7). Compared with that in the placebo and sorafenib nonresponder groups, the expression of TXNRD1, LCAT, and G6PD in the sorafenib responder 
239 group showed significant downregulation, and that of PTGES, RRM2, and ENTPD2 showed 240 significant upregulation. The expression of $U C K 2$ was statistically significant in the sorafenib 241 non-responder and responder groups, and was downregulated in the sorafenib responder group, 242 relative to the expression in the non-responder and placebo groups. The expression levels of 243 LPCAT1 and DTYMK were not significantly different between patients treated with sorafenib 244 (responders and non-responders) and those treated with the placebo. In other words, the MRGs in 245 the model showed a good response to sorafenib, which suggests the effectiveness of our MRG 246 signature in predicting the prognosis of patients with HCC.

\section{Nine MRGs for GSEA}

248 GSEA was conducted to ascertain significant changes in the potential pathways between high-

249

250

251

252

253

254

255

256

257

258

259

260

261

262

263

264

265

266

267

268

269

270

271

272

273

274

275

276

277

278

risk and low-risk populations. Based on the selection criteria of an $\mathrm{FDR}<0.05$, four significantly altered pathways in the high-risk group were observed: the p53 signalling pathway, the cell cycle pathway, purine metabolism, and pyrimidine metabolism (Fig. 8).

\section{Construction of nomograms}

A quantitative method was used, and a nomogram was constructed to predict the 1-, 3-, and 5year OS of patients with HCC in the discovery and validation cohorts, based on their common clinical traits (Fig. 9). The calibration curves revealed that the nomograms for the discovery and the validation cohorts had good accuracy, as observed in the ideal model.

\section{Discussion}

HCC accounts for more than $80 \%$ of liver cancer cases. It is a highly malignant, recurrent, and drug-resistant cancer that is often diagnosed at an advanced stage. Metabolic changes are widely reported characteristics of HCC (Grandhi et al. 2016). Although the three major liver metabolism pathways (glucose, lipid, and protein pathways) have been identified, these are far from enough to reveal the metabolic changes related to HCC (De Matteis et al. 2018; Lee et al. 2016; Liang et al. 2018; Shang et al. 2016; Tang et al. 2018). We constructed an MRG signature comprising nine genes, using TCGA data, and further verified its association with OS using an independent dataset to confirm the sensitivity and specificity of the model. The MRG signature had statistically significant prognostic value for male patients, those $\leq 65$ years, and those carrying wild-type TP53 or CTNNB1. Next, we performed independent drug verification of this signature using a GEO dataset, revealing that some of the identified genes showed good response to sorafenib. Overall, these results demonstrate the effectiveness of the MRG signature. Clinical findings have shown that the incidence of HCC and the associated mortality rates are higher in men than in women (Sukocheva 2018). This has been attributed to the lack of a protective effect of high oestrogen levels in men who drink heavily (Baecker et al. 2018; Montano-Loza et al. 2018; Singh et al. 2019). Although the pathogenesis of alcohol-induced HCC is complicated and still unclear, it is certain that alcohol toxicity causes liver cells to catabolise fatty acids, resulting in fat accumulation and fibrosis. Additionally, alcohol inhibits natural killer cells, which play key roles in antiviral, antitumour, and antifibrotic defence in terms of innate immunity. It also impairs the proteasome functions of macrophages and dendritic cells in terms of adaptive immunity, thus altering the presentation of alloantigens. Furthermore,

Peer) reviewing PDF | (2020:04:47605:2:1:NEW 28 Jul 2020) 
279 the oxidative metabolites of alcohol can interfere with DNA methylation, synthesis, and repair, 280 promote HCC carcinogenesis, and increase HCC sensitivity (Ceni et al. 2014; Miller et al. 2011). 281 In our study, we observed that in TCGA cohort, alcohol intake was significantly greater in 282 patients aged $\leq 65$ years than in patients aged $>65$ years, and the drinking history was longer in 283 the former. Furthermore, mutant alleles for TP53 and CTNNB1 were more frequent in men 284 (33.5\% and $34.7 \%$, respectively) than in women ( $21.8 \%$ and $12.7 \%$, respectively).

285 The MRG signature had a significant prognostic value for patients carrying wild-

286

287

288

289

290

291

292

293

294

295

296

297

298

299

300

301

302

303

304

305

306

307

308

309

310

311

312

313

314

315

316

317

318

type TP53 and CTNNB1 in both cohorts and also mutant TP53 and CTNNB1 in the ICGC cohort (Supplementary Figure 1), thus suggesting that TP53 and CTNNB1 are risk-indicator genes for $\mathrm{HCC}$, regardless of the presence or absence of mutations. More importantly, seven genes in the model showed good responsiveness to sorafenib. Studies by Lee et al. (Lee et al. 2019) showed that thioredoxin reductase 1 (TXNRD1) is significantly overexpressed in cytoplasmic subunits and is a key enzyme of the thioredoxin system, and is related to poor clinical pathological features and survival outcomes of HCC patients. Targeting TXNRDI results in accumulation of reactive oxygen species, causing cancer cells to experience high levels of oxidative stress. Auranofin, a TXNRD1 inhibitor, effectively exacerbates oxidative stress. This is consistent with our validation of sorafenib sensitivity using the GEO data set, which revealed that TXNRDI expression was significantly downregulated in the sorafenib responder group relative to that in the placebo and sorafenib non-responder groups. Considering the relatively small sample size in this study, the results must be validated in larger cohorts.

We used the common clinical traits of patients in TCGA and ICGC groups to construct a new nomogram, based on the expression of the nine MRGs, to accurately predict 1-, 3-, and 5-year survival rates. However, our sample sizes were limited, and only patients with complete information were included in the analysis, thereby limiting the precision of our estimates. However, we found that five (RRM2, TXNRD1, DTYMK, UCK2 and ENTPD2) of the nine MRGs were enriched in the purine metabolism and pyrimidine metabolism pathways, which was an important finding and indicated that the purine and pyrimidine metabolism pathways had a significant impact on the prognosis of HCC. Human ribonucleotide reductase (RR) comprised of RRM1 and RRM2 can maintain the steady state of the nucleotide library by converting ribonucleoside diphosphate to 2'-deoxyribonucleoside diphosphate, and increased expression and activity of RR are related to malignant transformation and growth; therefore, the key role of RR in DNA synthesis and repair makes it an important anticancer target. For example, the antiRRM2 siRNA duplex shows anti-proliferative activity in cancer cells. In addition, in vitro experiments verified that sorafenib inhibited the expression of RRM2 in HCC cells, which was positively correlated with the anticancer activity of sorafenib, proving that RRM2 is a new molecular target of sorafenib in HCC cells (Yang et al. 2020). Ectonucleoside triphosphate diphosphohydrolase 2 (ENTPD2) can be induced by hypoxia by stabilising hypoxia-inducible factor 1 (HIF-1) and its overexpression in clinical specimens of HCC. Myeloid-derived suppressor cells (MDSC) have immunosuppressive activity, which can enable cancer to evade immune surveillance and become unresponsive to immune checkpoint blockade. Hypoxia is the

Peer) reviewing PDF | (2020:04:47605:2:1:NEW 28 Jul 2020) 
cause of MDSC accumulation. ENTPD2 converts extracellular ATP to 5'-AMP, which prevents the differentiation of MDSC and promotes their maintenance. In this way, MDSC can promote tumour immune escape (Chiu et al. 2017). Uridine-cytidine kinase 2 (UCK2) is a pyrimidine ribonucleoside kinase that catalyses the phosphorylation of uridine and cytidine to UMP and CMP. The enzyme also catalyses the phosphorylation of several cytotoxic ribonucleoside analogues, and it has been studied as a possible chemotherapeutic agent for the treatment of cancer (Malami \& Abdul 2019; Murata et al. 2004). In vitro experiments show that UCK2 knockdown can inhibit cell migration, invasion, and proliferation, whereas overexpression of UCK2 has the opposite effect. Animal model experiments confirmed that knocking out UCK2 can inhibit tumour growth in vivo (Huang et al. 2019). However, the specific underlying mechanism needs to be further verified. As an important gene that controls dTTP biosynthesis and DNA replication, deoxythymidylate kinase $(D T Y M K)$ is necessary for all dividing cells. Studies have shown that DTYMK knockdown and LKB1 loss DTYMK are synthetically lethal, that is, excessive consumption of DTYMK below a critical threshold is lethal to all dividing cells, especially those carrying low levels of deoxynucleotide pools, such as tumour cells that maintain a rapid growth rate (Liu et al. 2013). LKB1/STK11 is the main regulator of cell metabolism and energy stress response. The best characterised target is AMP-activated protein kinase (AMPK). In the case of low cellular ATP levels, AMPK is activated and directly phosphorylated by LKB1. AMPK in turn regulates the use of nutrients through the phosphorylation of various substrates, thereby restoring energy homeostasis and controlling the absorption and metabolism of nutrients (Mihaylova \& Shaw 2011). LKB1/STK11 deficiency can lead to extensive defects in metabolic control. Primary cells and cancer cell lines lacking LKB1 are sensitive to nutritional deprivation and other types of metabolic stress as evidenced by this (Wingo et al. 2009). This is a direct evidence that DTYMK affects the occurrence and development of cancer through LKB1. Notably, the enrichment results of RRM2, DTYMK, UCK2, and ENTPD2 in GSEA were highly consistent with their corresponding KEGG pathways. TXNRD1 was enriched in the pyrimidine metabolism pathway in GSEA, but has not yet been included in the pyrimidine metabolism pathway in the KEGG database (Fig. 8C and Supplementary Figure 2). There may be potential mechanisms that have not yet been discovered, and this important observation may have been ignored by previous researchers. The risk score of our model was proved to be an independent prognostic factor of HCC and it allows accurate evaluation of HCC prognosis.

\section{Conclusions}

In summary, we have systematically demonstrated the prognostic value of our MRG signature for male patients, patients aged $\leq 65$ years, and patients carrying the wild-type TP53 and CTNNB1 genes. We have revealed the association between this gene signature and sorafenib responsiveness and explained the causes of the different responses among the groups. These findings provide new information regarding HCC prevention, diagnosis, and prognosis, and can be used in developing precision medicine approaches for individualised treatment.

\section{Acknowledgement}


359 We would like to thank Editage for English language editing.

360

361

362

363

364

365

366

367

368

369

370

371

372

373

374

375

376

377

378

379

380

381

382

383

384

385

386

387

388

389

390

391

392

393

394

395

396

397

398

\section{References}

Baecker A, Liu X, La Vecchia C, and Zhang ZF. 2018. Worldwide incidence of hepatocellular carcinoma cases attributable to major risk factors. Eur $J$ Cancer Prev 27:205-212 DOI 10.1097/CEJ.0000000000000428.

Berndt N, Eckstein J, Heucke N, Gajowski R, Stockmann M, Meierhofer D, and Holzhutter HG. 2019. Characterization of Lipid and Lipid Droplet Metabolism in Human HCC. Cells 8 DOI 10.3390/cells8050512.

Bhagyaraj E, Ahuja N, Kumar S, Tiwari D, Gupta S, Nanduri R, and Gupta P. 2019. TGFbeta induced chemoresistance in liver cancer is modulated by xenobiotic nuclear receptor PXR. Cell Cycle 18:3589-3602 DOI 10.1080/15384101.2019.1693120.

Budny A, Kozlowski P, Kaminska M, Jankiewicz M, Kolak A, Budny B, Budny W, Niemunis-Sawicka J, Szczypior G, Kurniawka B, and Burdan F. 2017. [Epidemiology and risk factors of hepatocellular carcinoma]. Pol Merkur Lekarski 43:133-139 DOI

Calderaro J, Couchy G, Imbeaud S, Amaddeo G, Letouze E, Blanc JF, Laurent C, Hajji Y, Azoulay D, Bioulac-Sage P, Nault JC, and Zucman-Rossi J. 2017. Histological subtypes of hepatocellular carcinoma are related to gene mutations and molecular tumour classification. $J$ Hepatol 67:727-738 DOI 10.1016/j.jhep.2017.05.014.

Cancer Genome Atlas Research Network. Electronic address wbe, and Cancer Genome Atlas Research N. 2017. Comprehensive and Integrative Genomic Characterization of Hepatocellular Carcinoma. Cell 169:1327-1341 e1323 DOI 10.1016/j.cell.2017.05.046.

Ceni E, Mello T, and Galli A. 2014. Pathogenesis of alcoholic liver disease: role of oxidative metabolism. World J Gastroenterol 20:17756-17772 DOI 10.3748/wjg.v20.i47.17756.

Chiu DK, Tse AP, Xu IM, Di Cui J, Lai RK, Li LL, Koh HY, Tsang FH, Wei LL, Wong CM, Ng IO, and Wong CC. 2017. Hypoxia inducible factor HIF-1 promotes myeloid-derived suppressor cells accumulation through ENTPD2/CD39L1 in hepatocellular carcinoma. Nat Commun 8:517 DOI 10.1038/s41467-017-00530-7.

De Matteis S, Ragusa A, Marisi G, De Domenico S, Casadei Gardini A, Bonafe M, and Giudetti AM. 2018. Aberrant Metabolism in Hepatocellular Carcinoma Provides Diagnostic and Therapeutic Opportunities. Oxid Med Cell Longev 2018:7512159 DOI $10.1155 / 2018 / 7512159$.

Farazi PA, and DePinho RA. 2006. Hepatocellular carcinoma pathogenesis: from genes to environment. Nat Rev Cancer 6:674-687 DOI 10.1038/nrc1934.

Grandhi MS, Kim AK, Ronnekleiv-Kelly SM, Kamel IR, Ghasebeh MA, and Pawlik TM. 2016. Hepatocellular carcinoma: From diagnosis to treatment. Surg Oncol 25:74-85 DOI 10.1016/j.suronc.2016.03.002.

Hilmi M, Neuzillet C, Calderaro J, Lafdil F, Pawlotsky JM, and Rousseau B. 2019. Angiogenesis and immune checkpoint inhibitors as therapies for hepatocellular carcinoma: current knowledge and future research directions. $J$ Immunother Cancer 7:333 DOI 10.1186/s40425-019-0824-5.

Peer) reviewing PDF | (2020:04:47605:2:1:NEW 28 Jul 2020) 
399

400

401

402

403

404

405

406

407

408

409

410

411

412

413

414

415

416

417

418

419

420

421

422

423

424

425

426

427

428

429

430

431

432

433

434

435

436

437

438

439

Hsu CS, Chao YC, Lin HH, Chen DS, and Kao JH. 2015. Systematic Review: Impact of Interferon-based Therapy on HCV-related Hepatocellular Carcinoma. Sci Rep 5:9954 DOI 10.1038/srep09954.

Huang Q, Li J, Xing J, Li W, Li H, Ke X, Zhang J, Ren T, Shang Y, Yang H, Jiang J, and Chen Z. 2014. CD147 promotes reprogramming of glucose metabolism and cell proliferation in HCC cells by inhibiting the p53-dependent signaling pathway. J Hepatol 61:859-866 DOI 10.1016/j.jhep.2014.04.035.

Huang S, Li J, Tam NL, Sun C, Hou Y, Hughes B, Wang Z, Zhou Q, He X, and Wu L. 2019. Uridine-cytidine kinase 2 upregulation predicts poor prognosis of hepatocellular carcinoma and is associated with cancer aggressiveness. Mol Carcinog 58:603-615 DOI 10.1002/mc.22954.

Kim J, Yu L, Chen W, Xu Y, Wu M, Todorova D, Tang Q, Feng B, Jiang L, He J, Chen G, Fu X, and Xu Y. 2019. Wild-Type p53 Promotes Cancer Metabolic Switch by Inducing PUMADependent Suppression of Oxidative Phosphorylation. Cancer Cell 35:191-203 e198 DOI 10.1016/j.ccell.2018.12.012.

Lee D, Xu IM, Chiu DK, Leibold J, Tse AP, Bao MH, Yuen VW, Chan CY, Lai RK, Chin DW, Chan DF, Cheung TT, Chok SH, Wong CM, Lowe SW, Ng IO, and Wong CC. 2019. Induction of Oxidative Stress Through Inhibition of Thioredoxin Reductase 1 Is an Effective Therapeutic Approach for Hepatocellular Carcinoma. Hepatology 69:1768-1786 DOI 10.1002/hep.30467.

Lee S, Mardinoglu A, Zhang C, Lee D, and Nielsen J. 2016. Dysregulated signaling hubs of liver lipid metabolism reveal hepatocellular carcinoma pathogenesis. Nucleic Acids Res 44:5529-5539 DOI 10.1093/nar/gkw462.

Levrero M, and Zucman-Rossi J. 2016. Mechanisms of HBV-induced hepatocellular carcinoma. J Hepatol 64:S84-S101 DOI 10.1016/j.jhep.2016.02.021.

Liang JQ, Teoh N, Xu L, Pok S, Li X, Chu ESH, Chiu J, Dong L, Arfianti E, Haigh WG, Yeh MM, Ioannou GN, Sung JJY, Farrell G, and Yu J. 2018. Dietary cholesterol promotes steatohepatitis related hepatocellular carcinoma through dysregulated metabolism and calcium signaling. Nat Commun 9:4490 DOI 10.1038/s41467-018-06931-6.

Liu Y, Marks K, Cowley GS, Carretero J, Liu Q, Nieland TJ, Xu C, Cohoon TJ, Gao P, Zhang Y, Chen Z, Altabef AB, Tchaicha JH, Wang X, Choe S, Driggers EM, Zhang J, Bailey ST, Sharpless NE, Hayes DN, Patel NM, Janne PA, Bardeesy N, Engelman JA, Manning BD, Shaw RJ, Asara JM, Scully R, Kimmelman A, Byers LA, Gibbons DL, Wistuba, II, Heymach JV, Kwiatkowski DJ, Kim WY, Kung AL, Gray NS, Root DE, Cantley LC, and Wong KK. 2013. Metabolic and functional genomic studies identify deoxythymidylate kinase as a target in LKB1-mutant lung cancer. Cancer Discov 3:870-879 DOI 10.1158/2159-8290.CD-13-0015.

Malami I, and Abdul AB. 2019. Involvement of the uridine cytidine kinase 2 enzyme in cancer cell death: A molecular crosstalk between the enzyme and cellular apoptosis induction. Biomed Pharmacother 109:1506-1510 DOI 10.1016/j.biopha.2018.10.200.

Mihaylova MM, and Shaw RJ. 2011. The AMPK signalling pathway coordinates cell growth, autophagy and metabolism. Nat Cell Biol 13:1016-1023 DOI 10.1038/ncb2329.

Peer) reviewing PDF | (2020:04:47605:2:1:NEW 28 Jul 2020) 
440

441

442

443

444

445

446

447

448

449

450

451

452

453

454

455

456

457

458

459

460

461

462

463

464

465

466

467

468

469

470

471

472

473

474

475

476

477

478

Miller AM, Horiguchi N, Jeong WI, Radaeva S, and Gao B. 2011. Molecular mechanisms of alcoholic liver disease: innate immunity and cytokines. Alcohol Clin Exp Res 35:787-793 DOI 10.1111/j.1530-0277.2010.01399.x.

Montano-Loza AJ, Mazurak VC, Ebadi M, Meza-Junco J, Sawyer MB, Baracos VE, and Kneteman N. 2018. Visceral adiposity increases risk for hepatocellular carcinoma in male patients with cirrhosis and recurrence after liver transplant. Hepatology 67:914-923 DOI 10.1002/hep.29578.

Murata D, Endo Y, Obata T, Sakamoto K, Syouji Y, Kadohira M, Matsuda A, and Sasaki T. 2004. A crucial role of uridine/cytidine kinase 2 in antitumor activity of 3 '-ethynyl nucleosides. Drug Metab Dispos 32:1178-1182 DOI 10.1124/dmd.104.000737.

Nakagawa H, Hayata Y, Kawamura S, Yamada T, Fujiwara N, and Koike K. 2018. Lipid Metabolic Reprogramming in Hepatocellular Carcinoma. Cancers (Basel) 10 DOI 10.3390/cancers 10110447 .

Njei B, Rotman Y, Ditah I, and Lim JK. 2015. Emerging trends in hepatocellular carcinoma incidence and mortality. Hepatology 61:191-199 DOI 10.1002/hep.27388.

Ogasawara S, Chiba T, Ooka Y, Suzuki E, Maeda T, Yokoyama M, Wakamatsu T, Inoue M, Saito T, Kobayashi K, Kiyono S, Nakamura M, Nakamoto S, Yasui S, Tawada A, Arai M, Kanda T, Maruyama H, Yokosuka O, and Kato N. 2018. Characteristics of patients with sorafenib-treated advanced hepatocellular carcinoma eligible for second-line treatment. Invest New Drugs 36:332-339 DOI 10.1007/s10637-017-0507-3.

Pinter M, and Peck-Radosavljevic M. 2018. Review article: systemic treatment of hepatocellular carcinoma. Aliment Pharmacol Ther 48:598-609 DOI 10.1111/apt.14913.

Pope ED, 3rd, Kimbrough EO, Vemireddy LP, Surapaneni PK, Copland JA, 3rd, and Mody K. 2019. Aberrant lipid metabolism as a therapeutic target in liver cancer. Expert Opin Ther Targets 23:473-483 DOI 10.1080/14728222.2019.1615883.

Rebouissou S, and Nault JC. 2020. Advances in molecular classification and precision oncology in hepatocellular carcinoma. $J$ Hepatol 72:215-229 DOI 10.1016/j.jhep.2019.08.017.

Schlachterman A, Craft WW, Jr., Hilgenfeldt E, Mitra A, and Cabrera R. 2015. Current and future treatments for hepatocellular carcinoma. World $J$ Gastroenterol 21:8478-8491 DOI 10.3748/wjg.v21.i28.8478.

Shang RZ, Qu SB, and Wang DS. 2016. Reprogramming of glucose metabolism in hepatocellular carcinoma: Progress and prospects. World J Gastroenterol 22:9933-9943 DOI 10.3748/wjg.v22.i45.9933.

Singal AG, and El-Serag HB. 2015. Hepatocellular Carcinoma From Epidemiology to Prevention: Translating Knowledge into Practice. Clin Gastroenterol Hepatol 13:2140-2151 DOI 10.1016/j.cgh.2015.08.014.

Singh SP, Panigrahi S, Mishra D, and Khatua CR. 2019. Alcohol-associated liver disease, not hepatitis B, is the major cause of cirrhosis in Asia. J Hepatol 70:1031-1032 DOI 10.1016/j.jhep.2019.01.003.

Peer) reviewing PDF | (2020:04:47605:2:1:NEW 28 Jul 2020) 
479

480

481

482

483

484

485

486

487

488

489

490

491

492

493

494

495

496

497

498

499

500

501

502

Steel JL, Nadeau K, Olek M, and Carr BI. 2007. Preliminary results of an individually tailored psychosocial intervention for patients with advanced hepatobiliary carcinoma. $J$ Psychosoc Oncol 25:19-42 DOI 10.1300/J077v25n03_02.

Sukocheva OA. 2018. Estrogen, estrogen receptors, and hepatocellular carcinoma: Are we there yet? World J Gastroenterol 24:1-4 DOI 10.3748/wjg.v24.i1.1.

Tai WT, Shiau CW, Chen HL, Liu CY, Lin CS, Cheng AL, Chen PJ, and Chen KF. 2013. Mcl-1-dependent activation of Beclin 1 mediates autophagic cell death induced by sorafenib and SC-59 in hepatocellular carcinoma cells. Cell Death Dis 4:e485 DOI 10.1038/cddis.2013.18.

Tang L, Zeng J, Geng P, Fang C, Wang Y, Sun M, Wang C, Wang J, Yin P, Hu C, Guo L, Yu J, Gao P, Li E, Zhuang Z, Xu G, and Liu Y. 2018. Global Metabolic Profiling Identifies a Pivotal Role of Proline and Hydroxyproline Metabolism in Supporting Hypoxic Response in Hepatocellular Carcinoma. Clin Cancer Res 24:474-485 DOI 10.1158/1078-0432.CCR-171707.

Wingo SN, Gallardo TD, Akbay EA, Liang MC, Contreras CM, Boren T, Shimamura T, Miller DS, Sharpless NE, Bardeesy N, Kwiatkowski DJ, Schorge JO, Wong KK, and Castrillon DH. 2009. Somatic LKB1 mutations promote cervical cancer progression. PLoS One 4:e5137 DOI 10.1371/journal.pone.0005137.

Yang PM, Lin LS, and Liu TP. 2020. Sorafenib Inhibits Ribonucleotide Reductase Regulatory Subunit M2 (RRM2) in Hepatocellular Carcinoma Cells. Biomolecules 10 DOI 10.3390/biom 10010117.

Zucman-Rossi J, Villanueva A, Nault JC, and Llovet JM. 2015. Genetic Landscape and Biomarkers of Hepatocellular Carcinoma. Gastroenterology 149:1226-1239 e1224 DOI 10.1053/j.gastro.2015.05.061.

503 
Figure 1

Risk score, reflecting overall survival, based on the metabolism-related-gene signature comprising nine genes, in the TCGA and ICGC cohorts.

(A-B) Risk-score distribution in the TCGA and ICGC cohorts. (C-D) The survival status of patients in the high- and low-risk groups in the TCGA and ICGC cohorts. (E-F) Heatmap of the expression of the nine MRGs in the high- and low-risk groups and the TCGA and ICGC cohorts.

A
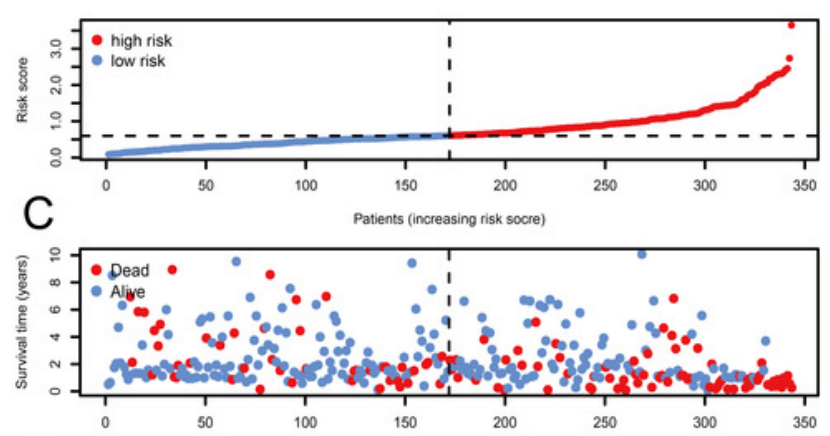

$\mathrm{E}$

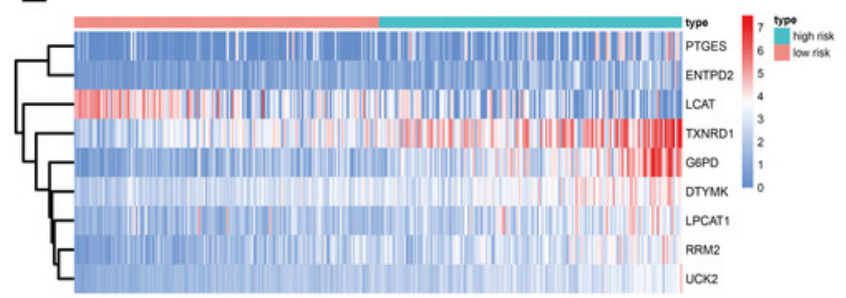

B ICGC Cohort

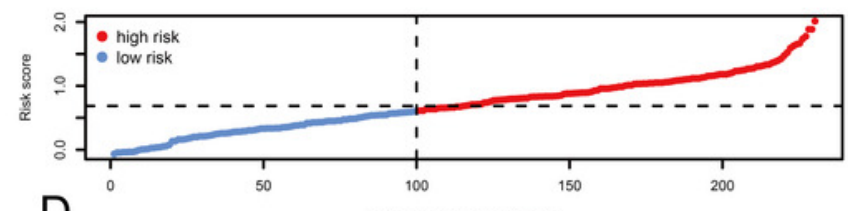

$\mathrm{D}$

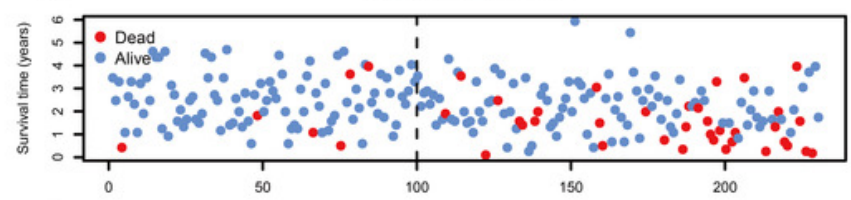

$\mathrm{F}$

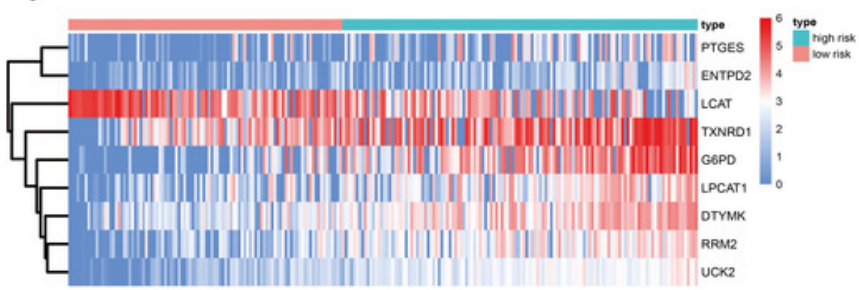


Figure 2

Validation of the risk scores based on the metabolism-related gene (MRG) signature comprising nine genes.

(A-B) Time-dependent receiver operating characteristic (ROC) curves of the MRG signature in the TCGA and ICGC cohorts. (C-D) Univariate Cox regression analysis for TCGA and ICGC cohorts. (E-F) Multivariate Cox regression analysis for TCGA and ICGC cohorts. 
A

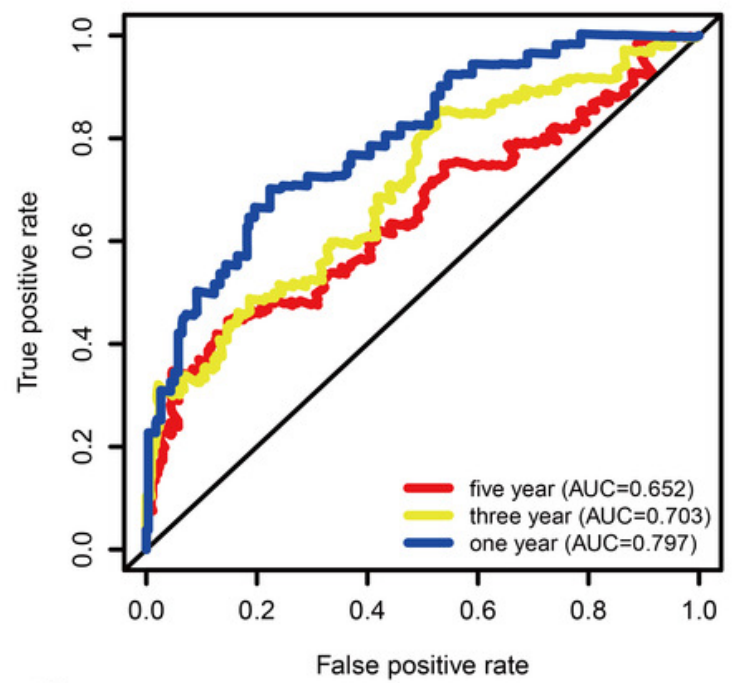

C

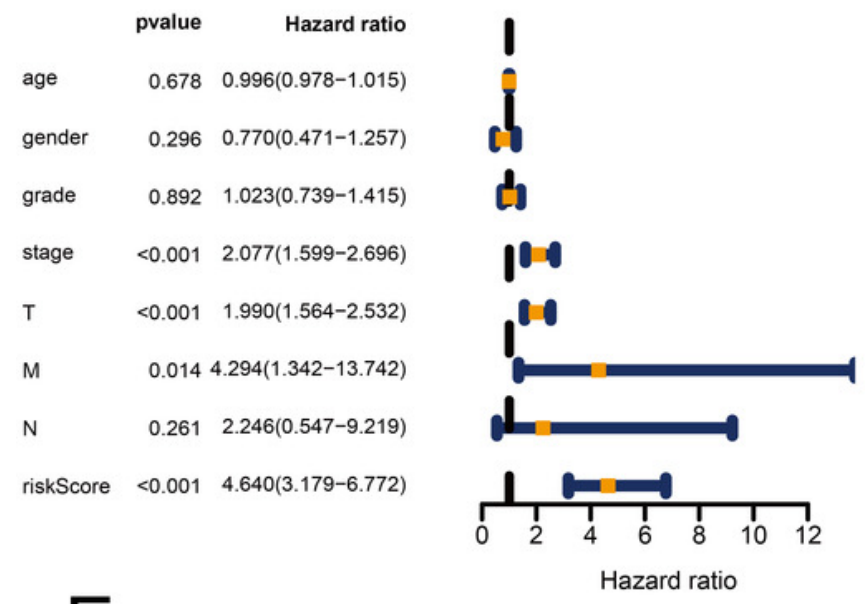

E

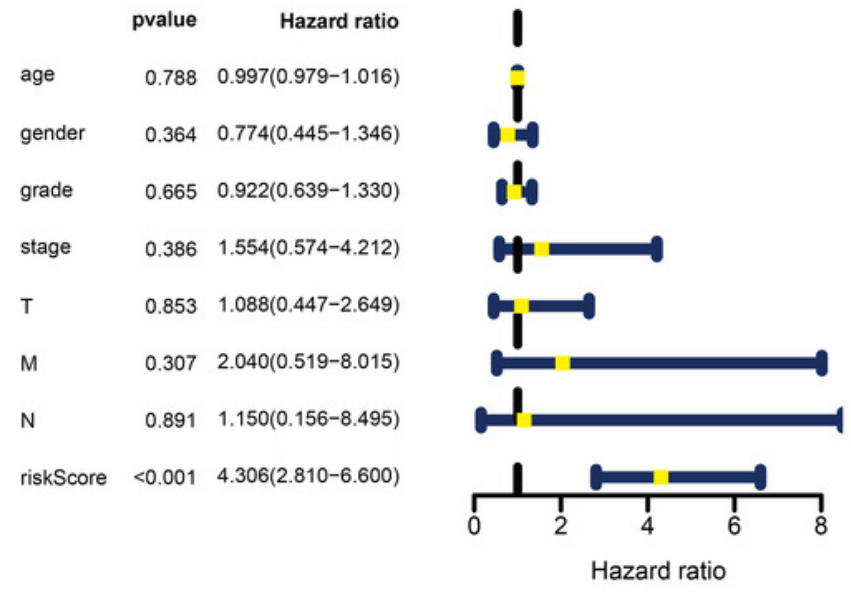

B ICGC OS
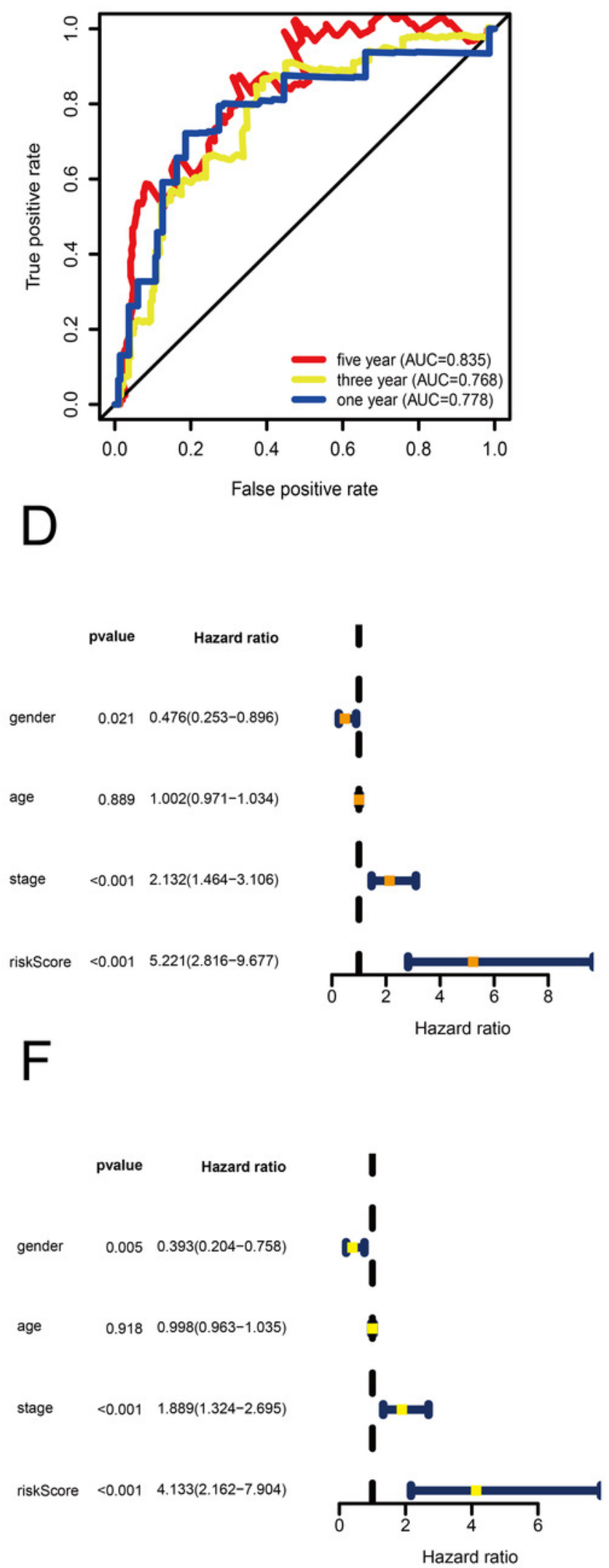
Figure 3

Survival probability based on the metabolism-related gene (MRG) signature comprising nine genes.

(A) Overall survival, OS; (B) relapse-free survival, RFS; and (C) progression-free survival, PFS, in the TCGA cohort. (D) OS in the ICGC cohort.

A

TCGA

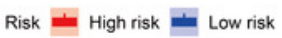

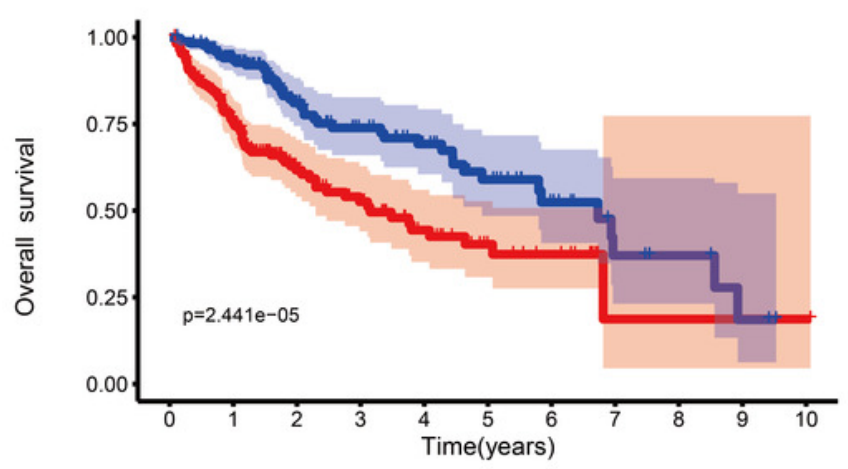

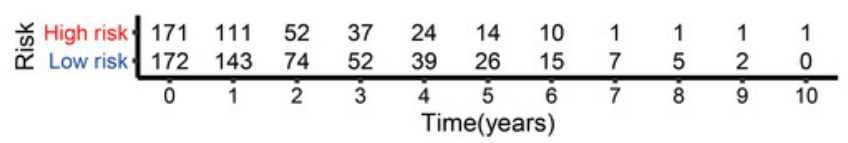

C

TCGA

Risk $=$ High risk

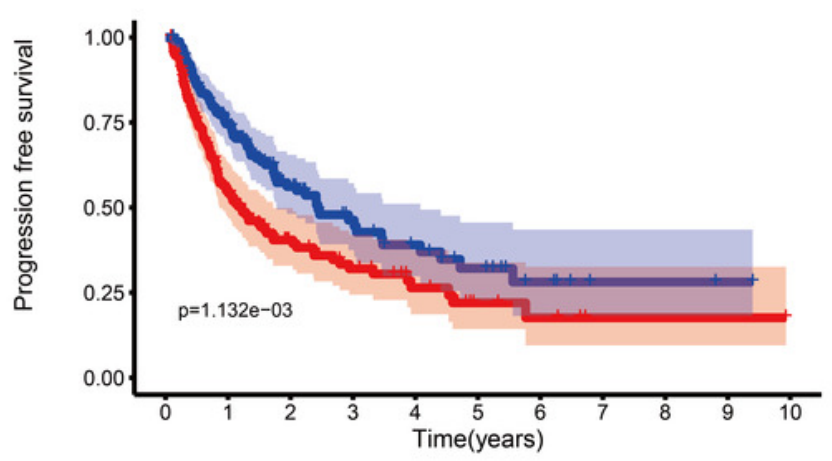

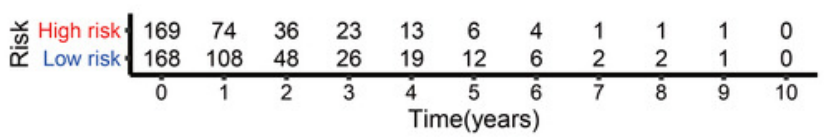

B

TCGA

Risk $=$ High risk $\perp$ Low risk

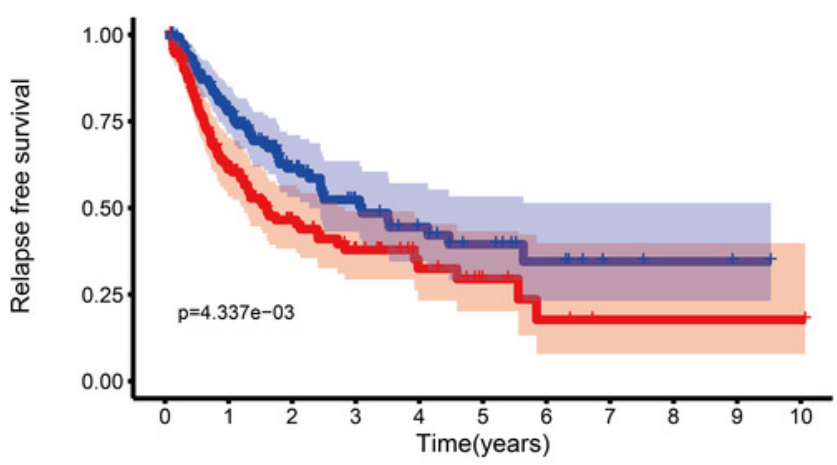

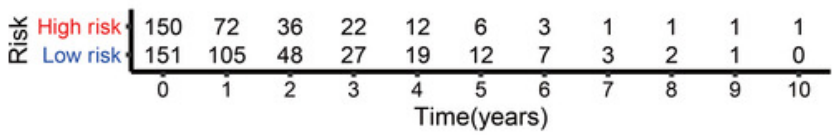

D

ICGC

Risk $\ldots$ High risk $\ldots$ Low risk

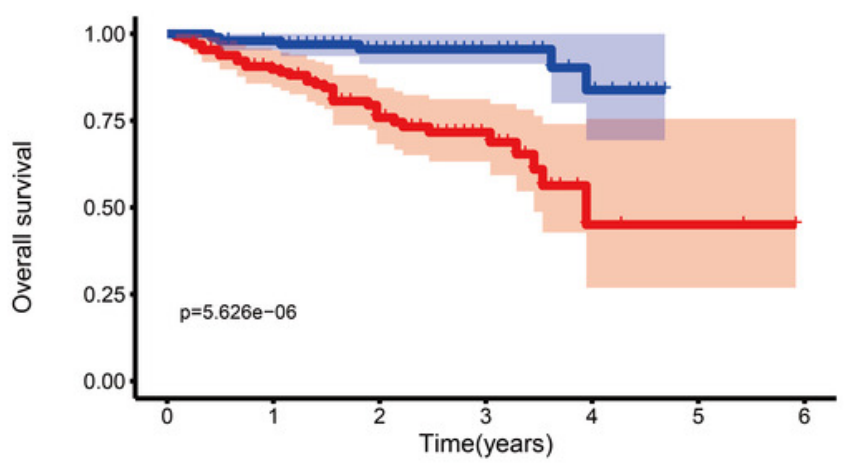

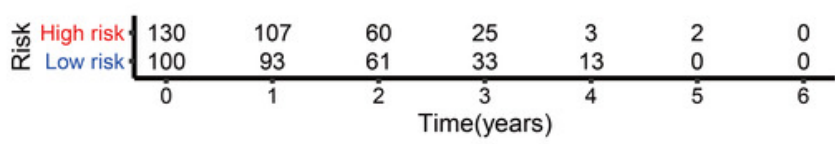


Figure 4

Association between survival and the metabolism-related gene (MRG) signature comprising nine genes, in male hepatocellular carcinoma patients.

(A) Overall survival, OS; (B) relapse-free survival, RFS; and (C) progression-free survival, PFS, in the TCGA cohort. (D) OS in the ICGC cohort.

A

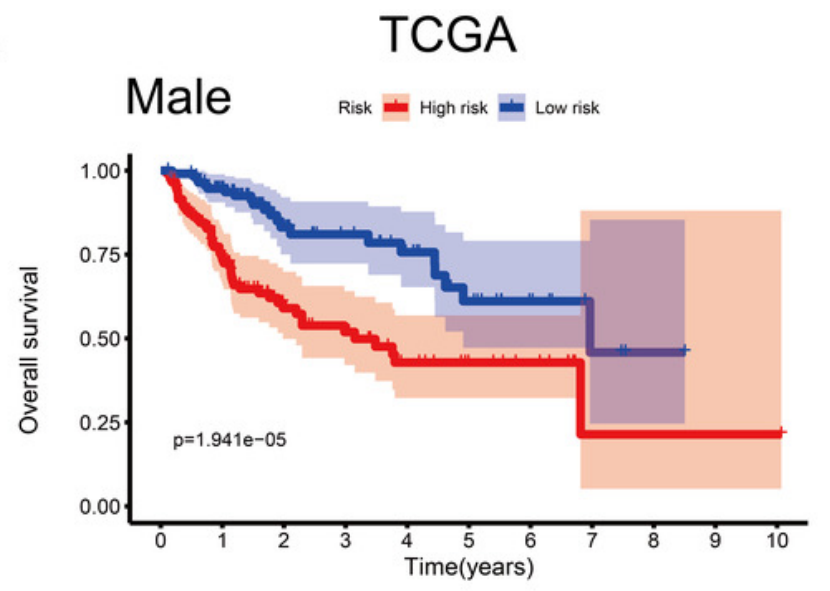

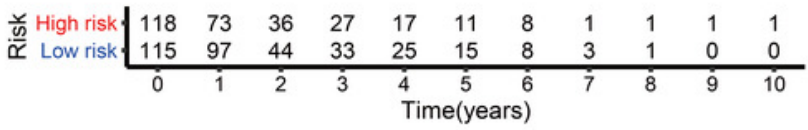

C

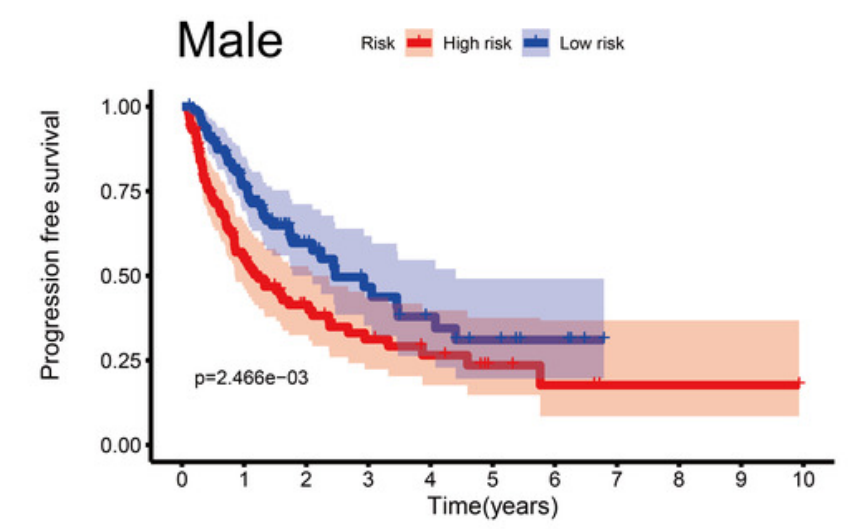

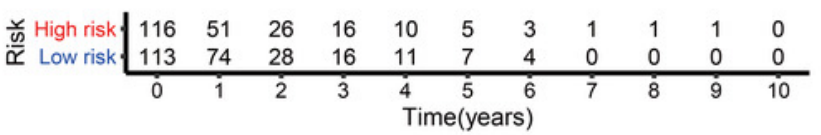

B

TCGA

Male Risk $=$ High risk $=$ Low isk

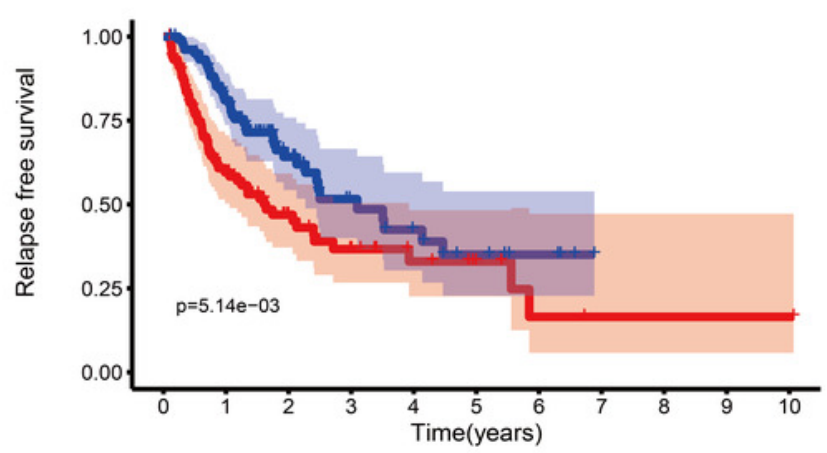

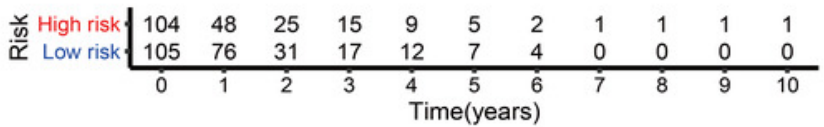

D ICGC

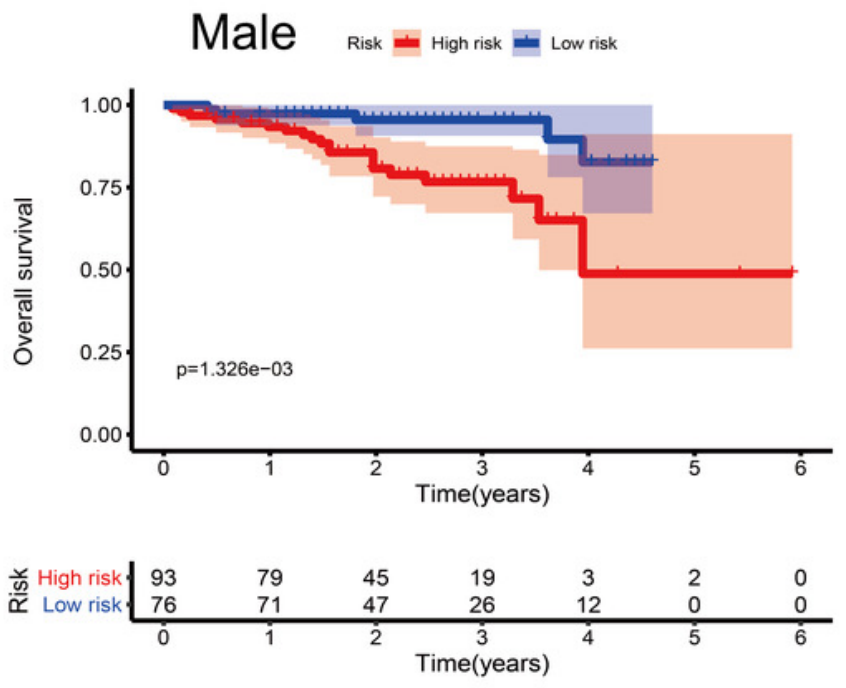


Figure 5

Association between survival probability and the metabolism-related gene (MRG) signature comprising nine genes, in hepatocellular carcinoma patients aged $\leq 65$ years.

(A) Overall survival, OS; (B) relapse-free survival, RFS; and (C) progression-free survival, PFS, in the TCGA cohort. (D) OS in the ICGC cohort.

A

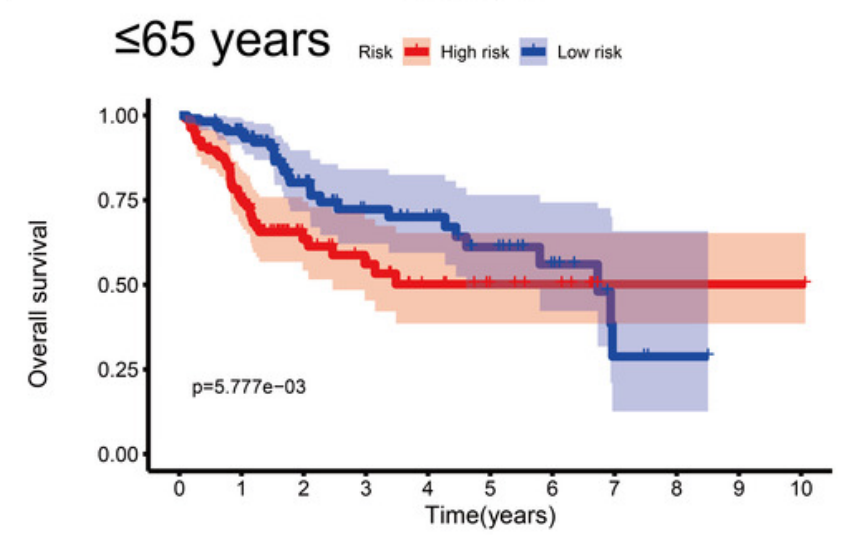

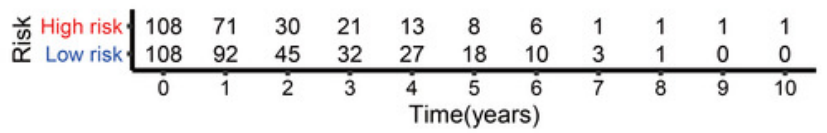

C

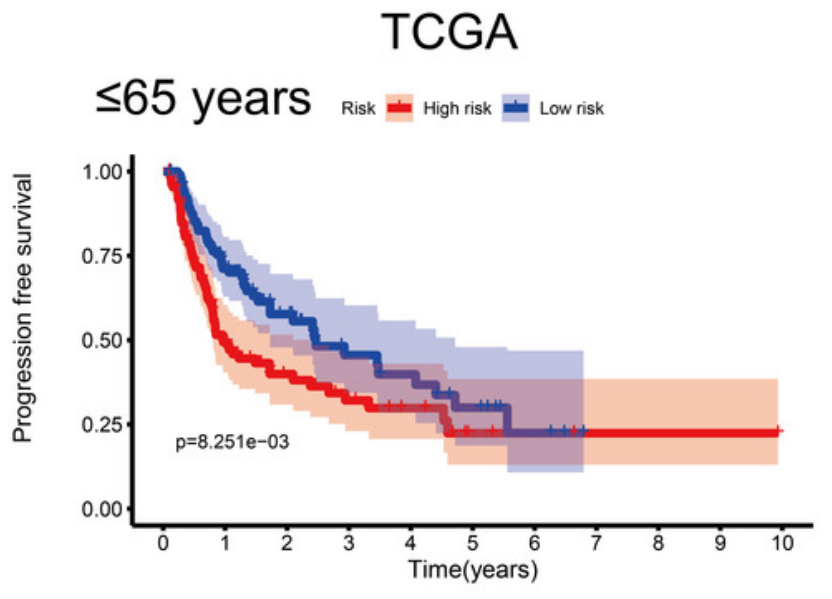

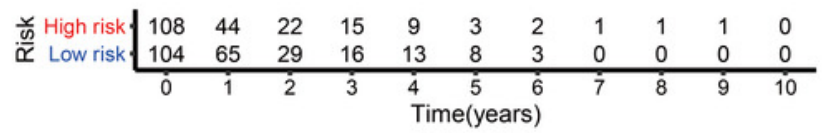

B $\leq 65$ years Risk ${ }_{\text {High }}$ isk

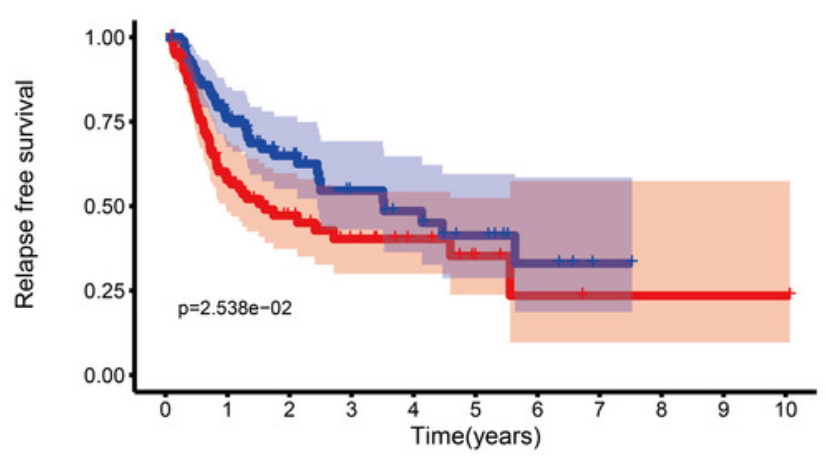

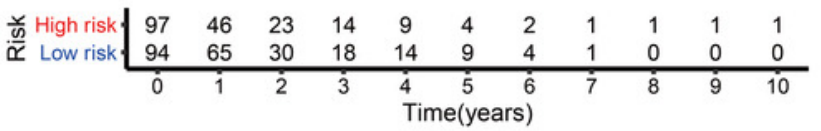

D ICGC

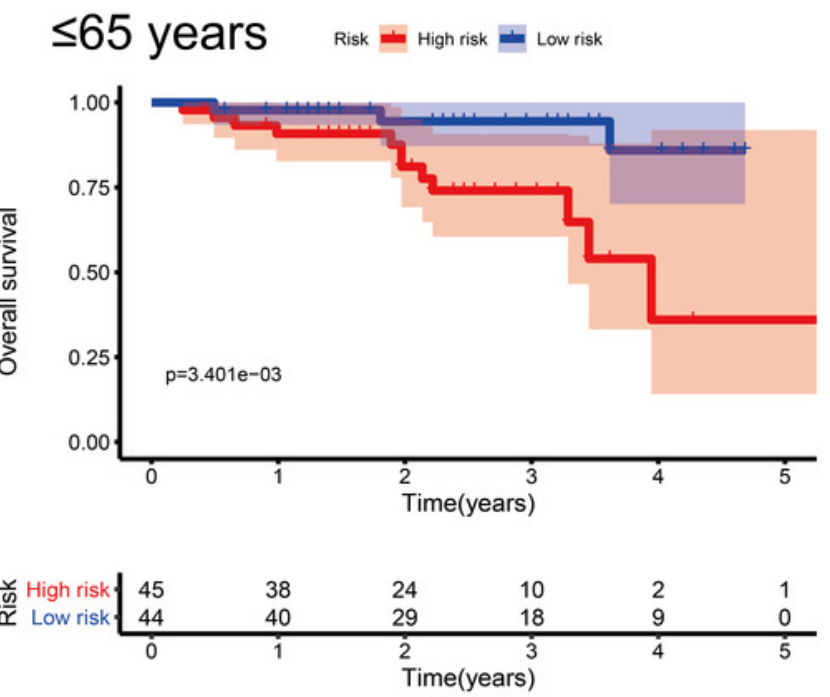


Figure 6

Kaplan-Meier curves depicting overall survival (OS) in hepatocellular carcinoma patients.

(A-B) Patients with wild-type TP53 in the TCGA and ICGC cohorts, respectively. (C-D) Patients with wild-type CTNNB1 in the TCGA and ICGC cohorts, respectively.

A

TCGA Cohort

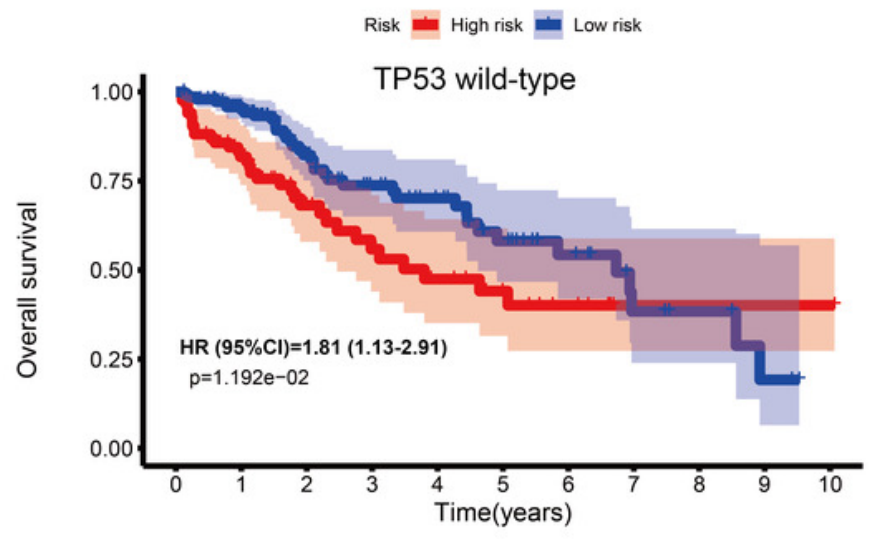

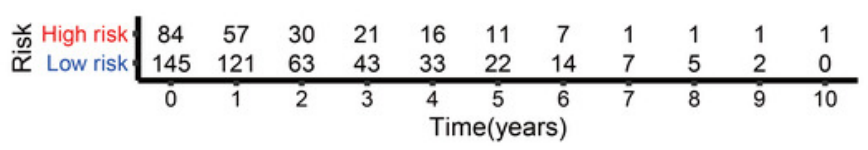

C

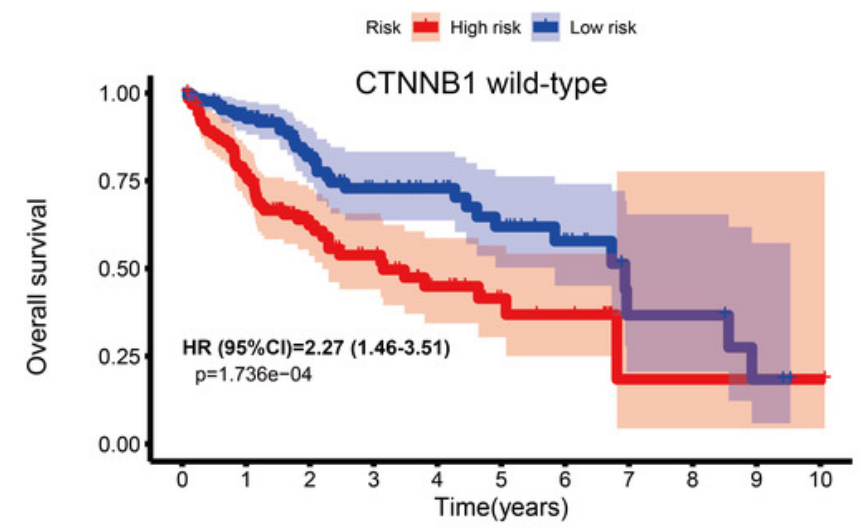

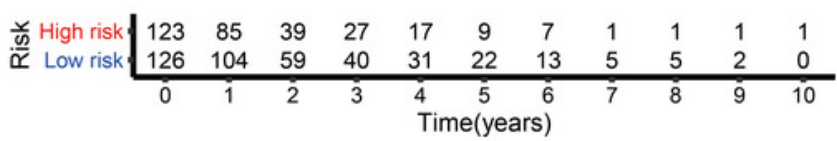

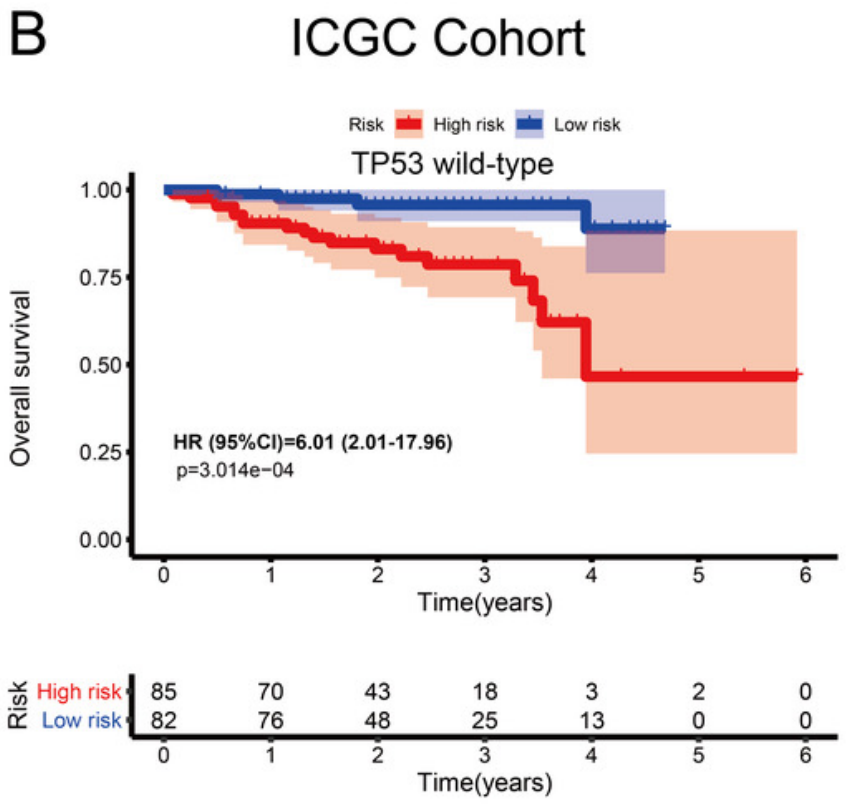

D

ICGC Cohort

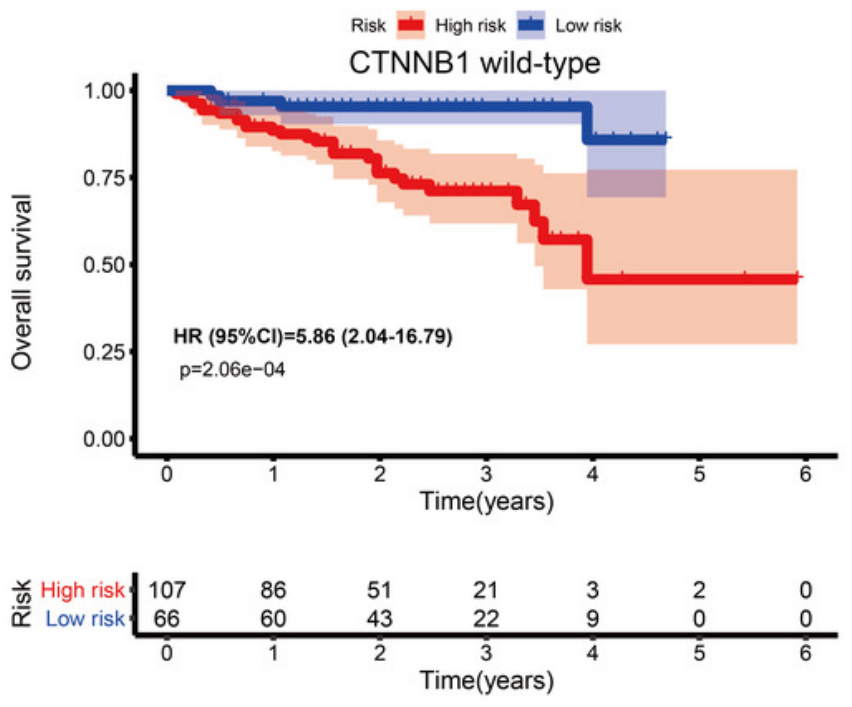


Figure 7

Sorafenib sensitivity validation for the nine metabolism-related genes (MRGs) in the independent cohort from the GEO database.

(A-I) Differences in the expression of these genes, between patients who received the placebo and sorafenib treatment, and between responders and non-responders. KruskalWallis test: ${ }^{* * *} P<0.001, * * P<0.01, * P<0.05$, ns: not significant.
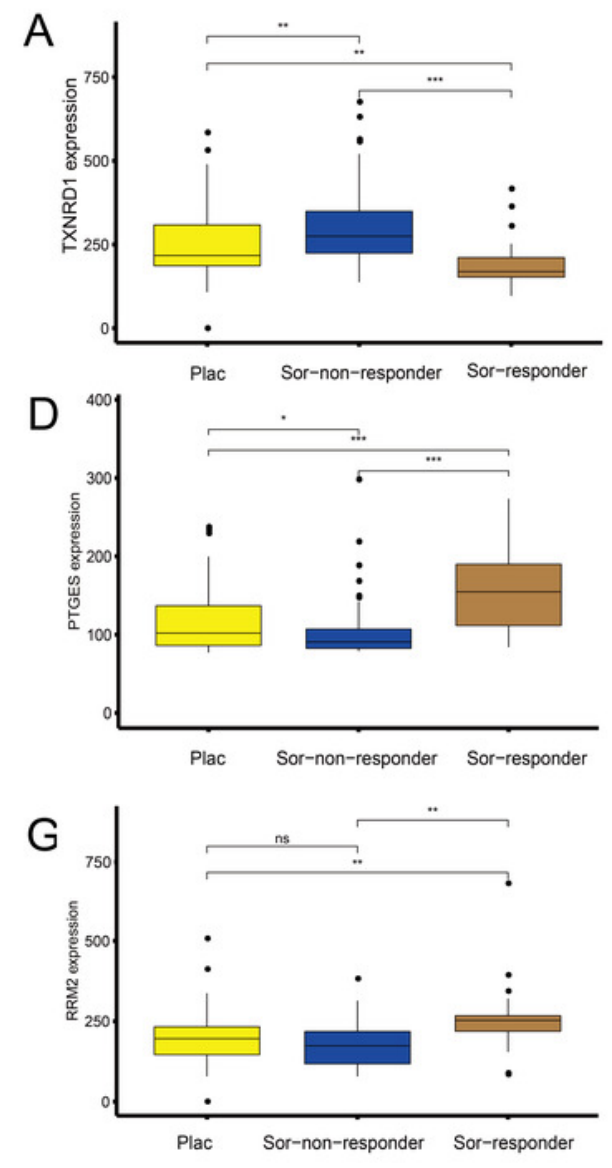

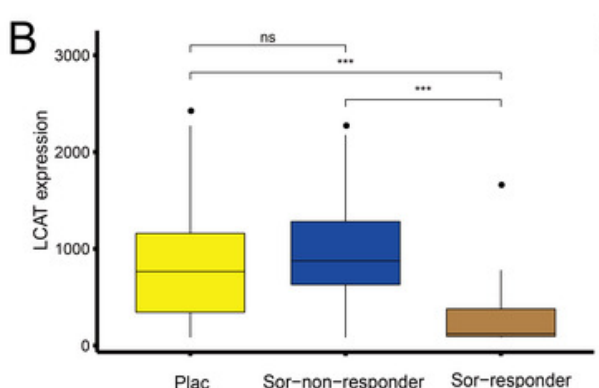

E
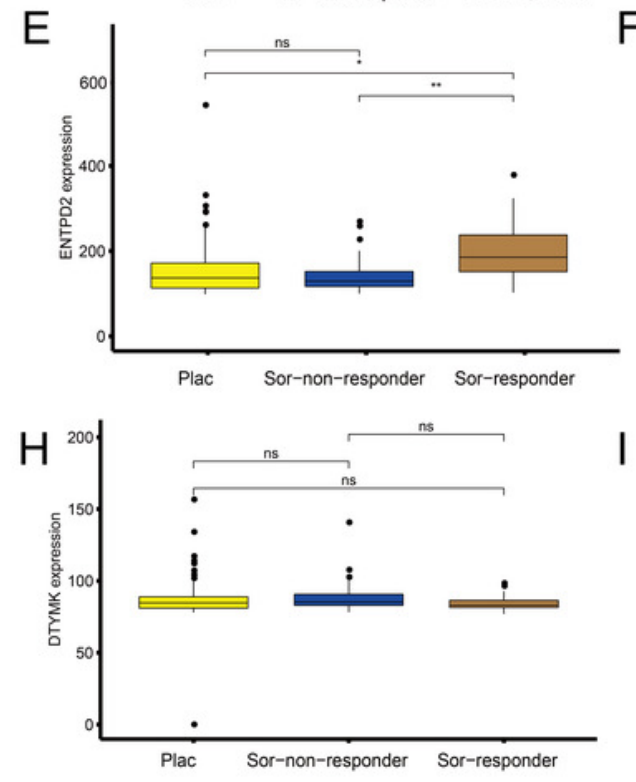

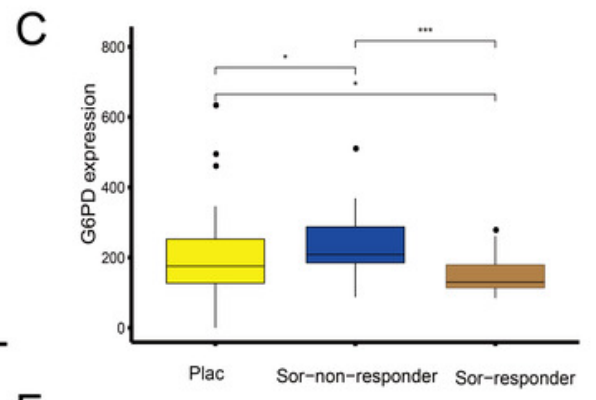

$\mathrm{F}$
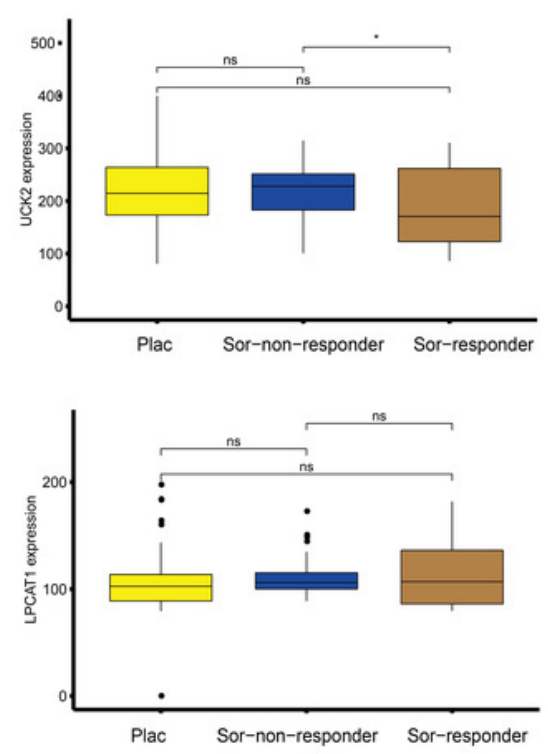
Figure 8

Gene set enrichment analysis (GSEA)

(A-B) Metabolism-related gene (MRG) signature comprising the nine genes that had a falsediscovery rate $<0.05$. (C) Circle diagram showing the gene components of four pathways. The large genes are those used in the MRG signature. 
A
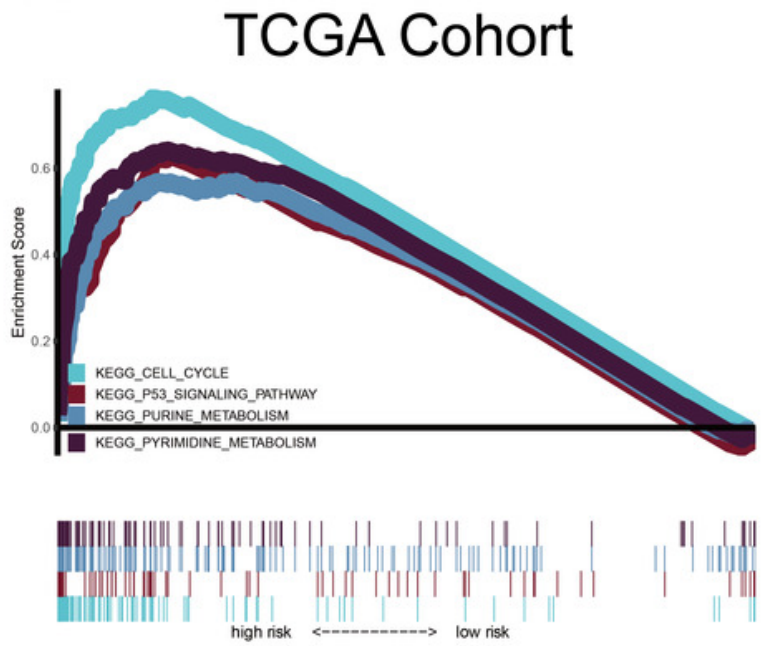

B
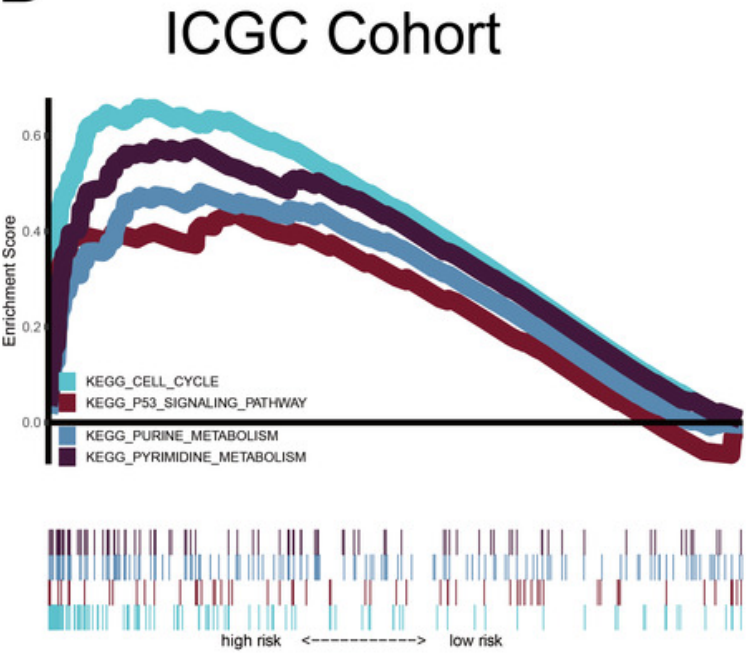

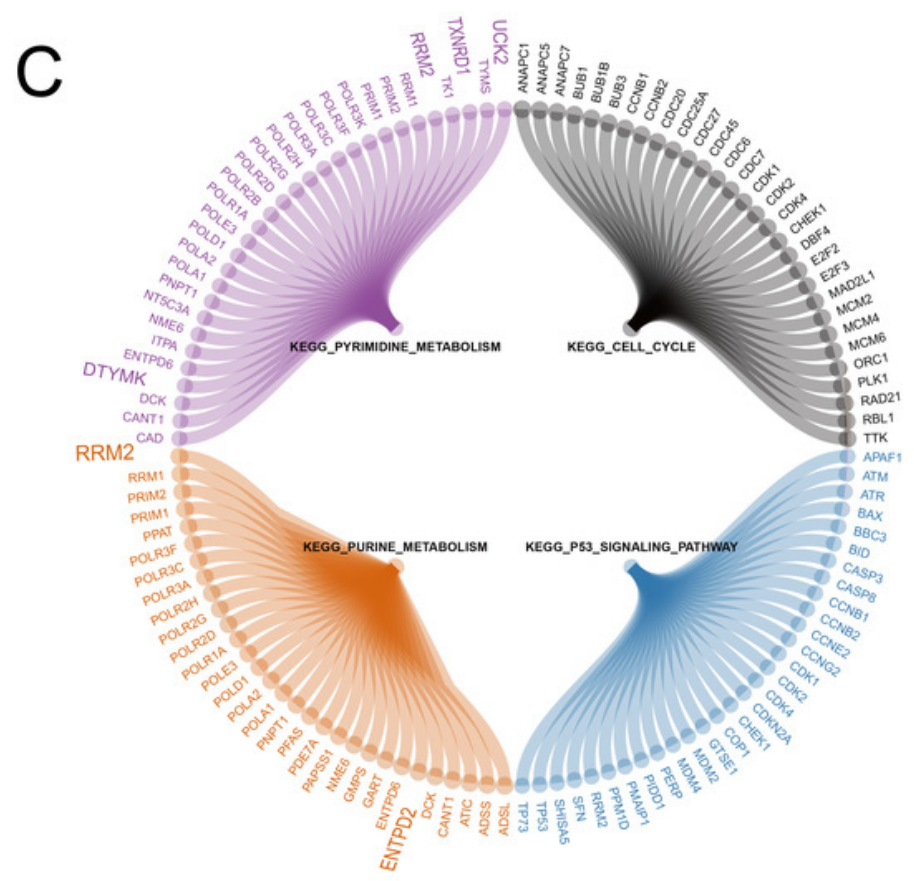


Figure 9

Nomograms to predict overall survival (OS) in hepatocellular carcinoma patients.

(A-B) Nomograms using clinical traits shared between the TCGA and ICGC cohorts.

Nomogram calibration curves for 1-, 3-, and 5-year OS, in the TCGA (C-E) and ICGC (F-H) cohorts. AJCC: American Joint Committee on Cancer; LCSGJ: Liver Cancer Study Group of Japan. 
A

TCGA Cohort

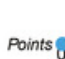

age

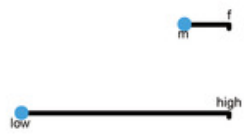

AJCC stage**.

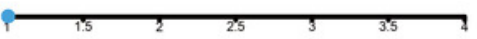

Total-points-to-outcome nomogram:

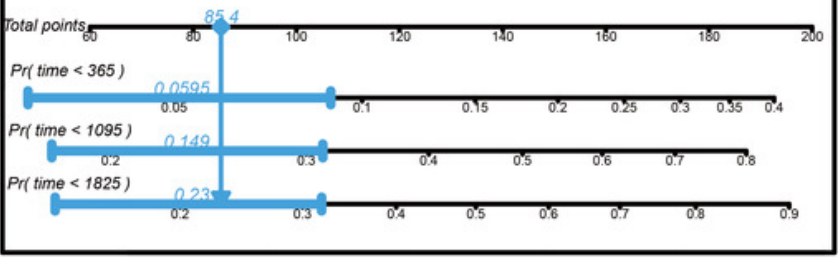

B ICGC Cohort

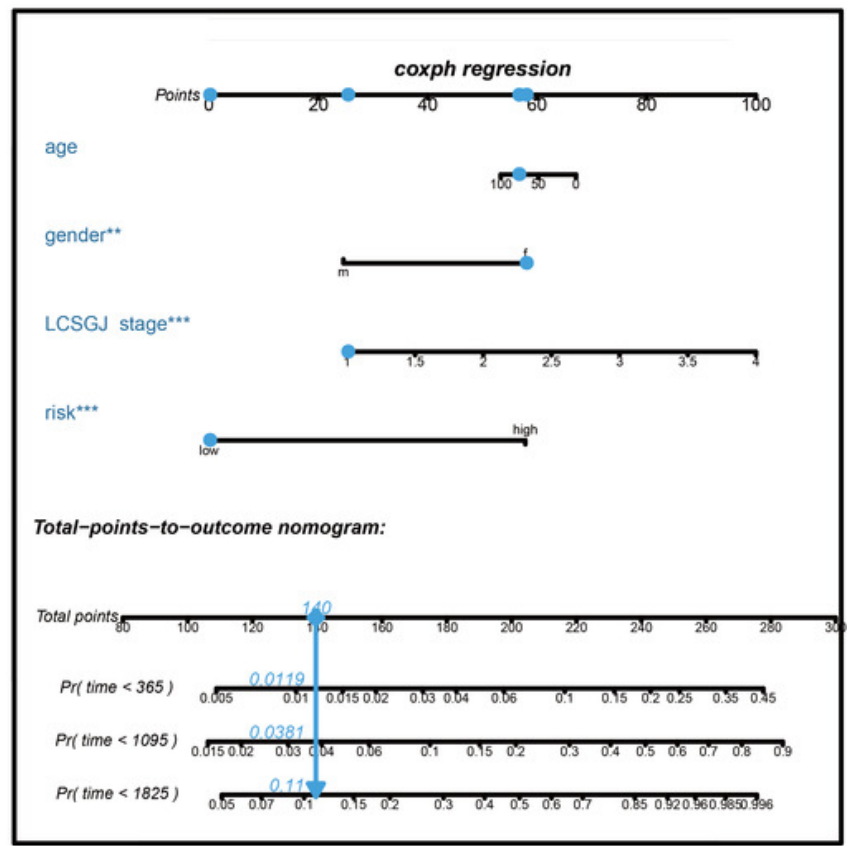

\section{TCGA 1 year}

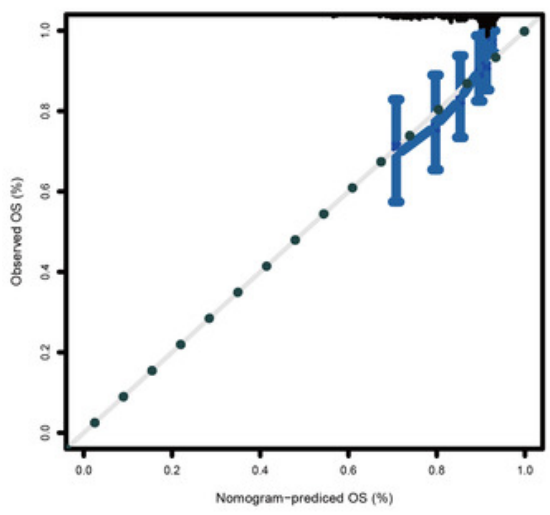

F ICGC 1 year

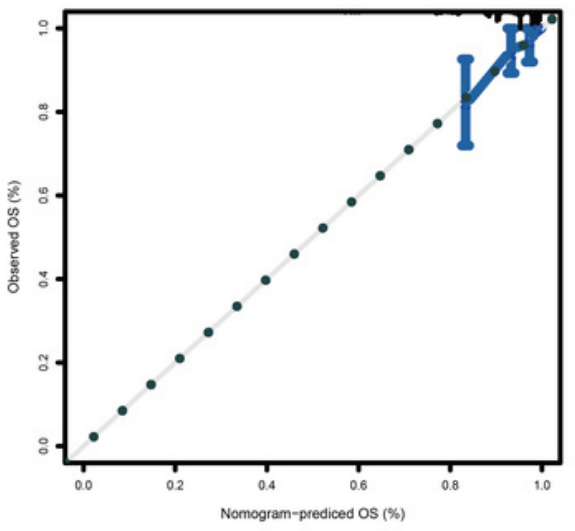

D TCGA 3 years

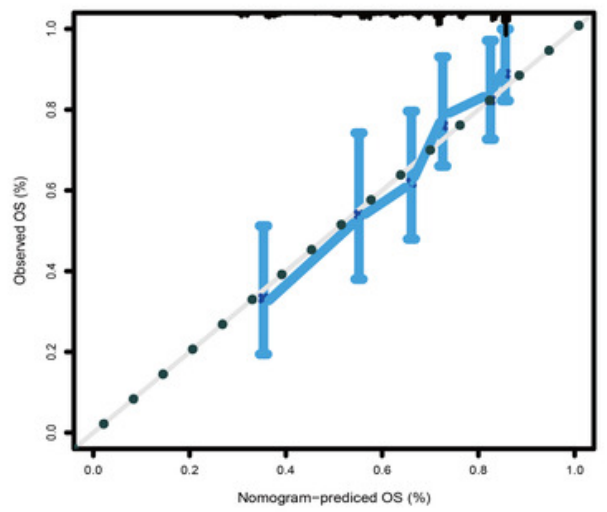

G ICGC 3 years

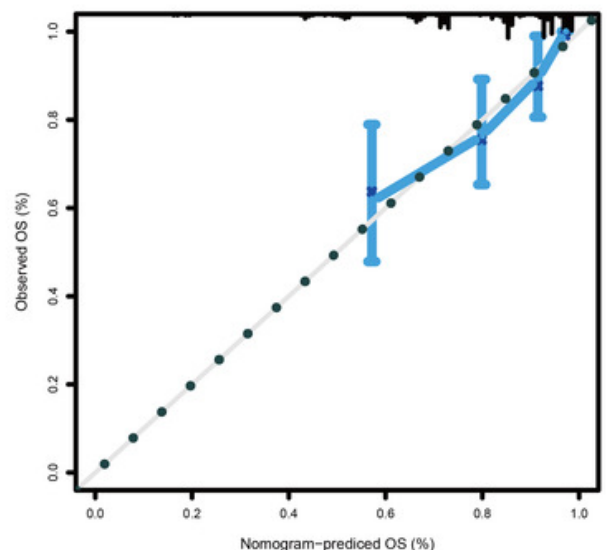

E TCGA 5 years

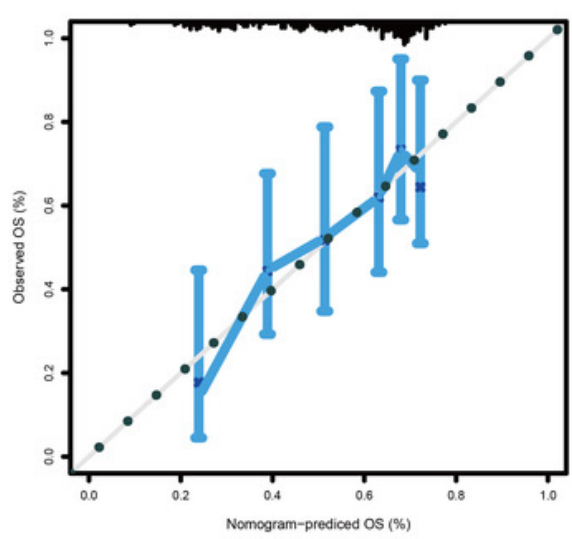

H ICGC 5 years

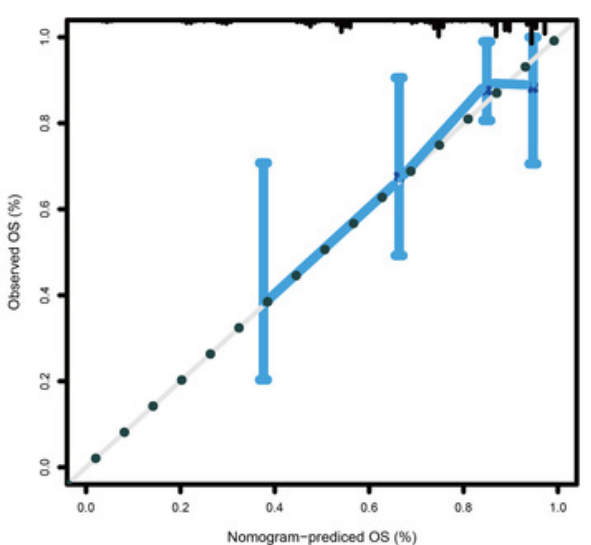




\section{Table 1 (on next page)}

Association between survival probability and the metabolism-related-gene signature comprising nine genes, in hepatocellular carcinoma patients in the TCGA cohort.

HR: hazard ratio; $\mathrm{Cl}$ : confidence interval; HBV: hepatitis B virus; HCV: hepatitis B virus; G: grade; T: tumor; AJCC: American Joint Committee on Cancer. 


\begin{tabular}{|c|c|c|c|c|c|c|c|c|c|}
\hline \multirow[b]{2}{*}{ Parameter } & \multicolumn{4}{|c|}{ OS cohort } & \multicolumn{3}{|l|}{ RFS cohort } & \multicolumn{2}{|l|}{ PFS cohort } \\
\hline & $\begin{array}{c}\text { Volume } \\
\text { (High/Low) }\end{array}$ & $\operatorname{HR}(95 \% \mathrm{CI})$ & $P$-value & $\begin{array}{c}\text { Volume } \\
\text { (High/Low) }\end{array}$ & $\begin{array}{c}\text { HR } \\
(95 \% \mathrm{CI})\end{array}$ & $P$-value & $\begin{array}{c}\text { Volume } \\
\text { (High/Low) }\end{array}$ & $\begin{array}{c}\mathrm{HR} \\
(95 \% \mathrm{CI})\end{array}$ & $P$-value \\
\hline Total & $171 / 172$ & $\begin{array}{c}2.20 \\
(1.51-3.19)\end{array}$ & $<0.001$ & $150 / 151$ & $\begin{array}{c}1.62 \\
(1.16-2.27)\end{array}$ & 0.005 & $169 / 168$ & $\begin{array}{c}1.64 \\
(1.21-2.20)\end{array}$ & 0.001 \\
\hline \multicolumn{10}{|l|}{ Age } \\
\hline$\leq 65$ & $108 / 108$ & $\begin{array}{c}1.97 \\
(1.21-3.21)\end{array}$ & 0.007 & 97/94 & $\begin{array}{c}1.62 \\
(1.06-2.47)\end{array}$ & 0.027 & $108 / 104$ & $\begin{array}{c}1.64 \\
(1.13-2.39)\end{array}$ & 0.009 \\
\hline$>65$ & $63 / 64$ & $\begin{array}{c}2.60 \\
(1.42-4.73)\end{array}$ & 0.002 & $53 / 57$ & $\begin{array}{c}1.61 \\
(0.93-2.77)\end{array}$ & 0.088 & $61 / 64$ & $\begin{array}{c}1.62 \\
(0.98-2.67)\end{array}$ & 0.061 \\
\hline \multicolumn{10}{|l|}{ Gender } \\
\hline Male & $118 / 115$ & $\begin{array}{c}2.85 \\
(1.73-4.71)\end{array}$ & $<\mathbf{0 . 0 0 1}$ & $104 / 105$ & $\begin{array}{c}1.77 \\
(1.18-2.66)\end{array}$ & 0.006 & $116 / 113$ & $\begin{array}{c}1.76(1.21- \\
2.55)\end{array}$ & 0.003 \\
\hline Female & $53 / 57$ & $\begin{array}{c}1.77 \\
(0.96-3.27)\end{array}$ & 0.069 & $46 / 46$ & $\begin{array}{c}1.34(0.74- \\
2.41)\end{array}$ & 0.334 & $53 / 55$ & $\begin{array}{c}1.42(0.85- \\
2.38)\end{array}$ & 0.181 \\
\hline $\begin{array}{l}\text { Risk } \\
\text { factors }\end{array}$ & & & & & & & & & \\
\hline
\end{tabular}




\begin{tabular}{|c|c|c|c|c|c|c|c|c|c|}
\hline $\begin{array}{l}\mathrm{HBV}+ \\
\mathrm{HCV}\end{array}$ & $67 / 75$ & $\begin{array}{c}2.24 \\
(1.08-4.69)\end{array}$ & 0.031 & $59 / 65$ & $\begin{array}{c}1.08 \\
(0.62-1.88)\end{array}$ & 0.792 & $69 / 72$ & $\begin{array}{c}1.32 \\
(0.83-2.10)\end{array}$ & 0.242 \\
\hline $\begin{array}{l}\text { Alcohol } \\
\text { intake }\end{array}$ & $56 / 55$ & $\begin{array}{c}1.93 \\
(1.00-3.71)\end{array}$ & 0.049 & $53 / 47$ & $\begin{array}{c}2.15 \\
(1.17-3.95)\end{array}$ & 0.014 & $57 / 53$ & $\begin{array}{c}1.84 \\
(1.08-3.14)\end{array}$ & 0.025 \\
\hline \multicolumn{10}{|l|}{$\begin{array}{l}\text { Degree of } \\
\text { fibrosis }\end{array}$} \\
\hline $\begin{array}{l}\text { No } \\
\text { fibrosi } \\
\text { S }\end{array}$ & $30 / 42$ & $\begin{array}{c}5.89 \\
(2.82- \\
12.28)\end{array}$ & $<0.001$ & $25 / 36$ & $\begin{array}{c}3.67 \\
(1.56- \\
8.66)\end{array}$ & 0.003 & $29 / 42$ & $\begin{array}{c}2.45 \\
(1.34- \\
4.50)\end{array}$ & 0.004 \\
\hline $\begin{array}{l}\text { fibrosi } \\
s \text { and } \\
\text { cirrhos } \\
\text { is }\end{array}$ & $60 / 67$ & $\begin{array}{c}3.55 \\
(1.74- \\
7.27)\end{array}$ & $<0.001$ & $53 / 58$ & $\begin{array}{c}2.50 \\
(1.18- \\
5.30)\end{array}$ & 0.017 & $61 / 63$ & $\begin{array}{c}2.55 \\
(1.40- \\
4.64)\end{array}$ & 0.002 \\
\hline \multicolumn{10}{|l|}{ Grade } \\
\hline G1-G2 & $83 / 131$ & $\begin{array}{c}2.20 \\
(1.38-3.49)\end{array}$ & $<0.001$ & $78 / 113$ & $\begin{array}{c}1.78 \\
(1.16-2.74)\end{array}$ & 0.008 & $81 / 128$ & $\begin{array}{c}1.67 \\
(1.13-2.46)\end{array}$ & 0.010 \\
\hline G3-G4 & $86 / 38$ & $\begin{array}{c}2.42 \\
(1.12-5.24)\end{array}$ & 0.025 & $70 / 35$ & $\begin{array}{c}1.54 \\
(0.83-2.87)\end{array}$ & 0.170 & $86 / 37$ & $\begin{array}{c}1.68 \\
(0.96-2.95)\end{array}$ & 0.071 \\
\hline $\begin{array}{l}\mathrm{T} \\
\text { classificati } \\
\text { on }\end{array}$ & & & & & & & & & \\
\hline
\end{tabular}




\begin{tabular}{|c|c|c|c|c|c|c|c|c|c|}
\hline T1-T2 & $120 / 132$ & $\begin{array}{c}2.44 \\
(1.45-4.11)\end{array}$ & $<0.001$ & $103 / 116$ & $\begin{array}{c}1.81 \\
(1.17-2.77)\end{array}$ & 0.007 & $120 / 128$ & $\begin{array}{c}1.94 \\
(1.34-2.81)\end{array}$ & $<0.001$ \\
\hline $\mathrm{T} 3-\mathrm{T} 4$ & $51 / 37$ & $\begin{array}{c}1.90 \\
(1.08-3.35)\end{array}$ & 0.025 & $47 / 32$ & $\begin{array}{c}1.21 \\
(0.70-2.10)\end{array}$ & 0.488 & $49 / 37$ & $\begin{array}{c}1.06 \\
(0.63-1.78)\end{array}$ & 0.823 \\
\hline \multicolumn{10}{|l|}{$\begin{array}{l}\text { AJCC } \\
\text { stage }\end{array}$} \\
\hline $\begin{array}{l}\text { Stage } \\
\text { I }\end{array}$ & $63 / 98$ & $\begin{array}{c}2.24 \\
(1.13-4.41)\end{array}$ & 0.020 & $52 / 86$ & $\begin{array}{c}1.43 \\
(0.80-2.58)\end{array}$ & 0.230 & $62 / 96$ & $\begin{array}{c}1.53 \\
(0.92-2.53)\end{array}$ & 0.099 \\
\hline $\begin{array}{l}\text { Stage } \\
\text { II }\end{array}$ & $47 / 30$ & $\begin{array}{c}2.55 \\
(0.94-6.95)\end{array}$ & 0.066 & $42 / 27$ & $\begin{array}{c}2.05 \\
(0.98-4.31)\end{array}$ & 0.057 & $47 / 28$ & $\begin{array}{c}2.09 \\
(1.07-4.09)\end{array}$ & 0.031 \\
\hline $\begin{array}{l}\text { Stage } \\
\text { III and } \\
\text { IV }\end{array}$ & $49 / 34$ & $\begin{array}{c}1.86 \\
(1.02-3.39)\end{array}$ & 0.043 & $45 / 29$ & $\begin{array}{c}1.21 \\
(0.68-2.14)\end{array}$ & 0.513 & $49 / 34$ & $\begin{array}{c}1.04 \\
(0.61-1.78)\end{array}$ & 0.881 \\
\hline
\end{tabular}




\section{Table 2 (on next page)}

Association between overall survival and the metabolism-related-gene signature comprising nine genes, in hepatocellular carcinoma patients in the ICGC cohort.

HR: hazard ratio; Cl: confidence interval; LCSGJ: Liver Cancer Study Group of Japan. 


\begin{tabular}{|c|c|c|c|}
\hline \multirow{3}{*}{ Parameter } & \multicolumn{3}{|c|}{ ICGC cohort } \\
\hline & Number & $\operatorname{HR}(95 \% \mathrm{CI})$ & $P$-value \\
\hline & (High/Lo & & \\
\hline Total & $130 / 100$ & $5.22(2.82-9.68)$ & $<0.001$ \\
\hline \multicolumn{4}{|l|}{ Age } \\
\hline$\leq 65$ & $45 / 44$ & $5.58(1.55-20.11)$ & 0.009 \\
\hline$>65$ & $85 / 56$ & $6.47(1.93-21.72)$ & 0.002 \\
\hline \multicolumn{4}{|l|}{ Gender } \\
\hline Male & $93 / 76$ & $4.51(1.66-12.25)$ & 0.003 \\
\hline Female & $37 / 24$ & $12.41(1.64-94.14)$ & 0.015 \\
\hline \multicolumn{4}{|c|}{ LCSGJ stage } \\
\hline Stage I & $22 / 15$ & $1.00(0.82-1.22)$ & 0.999 \\
\hline Stage II & $59 / 41$ & $2.68(0.98-7.36)$ & 0.056 \\
\hline
\end{tabular}


Stage III and IV $\quad 51 / 45$

$17.68(2.31-135.11) \quad 0.006$ 